October, 2007

\title{
On superconformal projective hypermultiplets
}

\author{
Sergei M. Kuzenko1 \\ School of Physics M013, The University of Western Australia \\ 35 Stirling Highway, Crawley W.A. 6009, Australia
}

\begin{abstract}
Building on the five-dimensional constructions in hep-th/0601177, we provide a unified description of four-dimensional $\mathcal{N}=2$ superconformal off-shell multiplets in projective superspace, including a realization in terms of $\mathcal{N}=1$ superfields. In particular, superconformal polar multiplets are consistently defined for the first time. We present new $4 \mathrm{D} \mathcal{N}=2$ superconformal sigma-models described by polar multiplets. Such sigma-models realize general superconformal couplings in projective superspace, but involve an infinite tale of auxiliary $\mathcal{N}=1$ superfields. The auxiliaries should be eliminated by solving infinitely many algebraic nonlinear equations, and this is a nontrivial technical problem. We argue that the latter can be avoided by making use of supersymmetry considerations. All information about the resulting superconformal model (and hence the associated superconformal cone) is encoded in the so-called canonical coordinate system for a Kähler metric, which was introduced by Bochner and Calabi in the late 1940s.
\end{abstract}

\footnotetext{
${ }^{1}$ kuzenko@cyllene.uwa.edu.au
} 


\section{Contents}

1 Introduction

2

$24 \mathrm{D} \mathcal{N}=2$ superconformal formalism

2.1 Superconformal Killing vectors . . . . . . . . . . . . . . . . 4

2.2 Superconformal projective multiplets: Definition . . . . . . . . . . . 6 6

2.3 Superconformal projective multiplets: Examples . . . . . . . . . . . 8 8

2.4 Superconformal action ............................. 11

2.5 Projective gauge fixing . . . . . . . . . . . . . . . 12

$34 \mathrm{D} \mathcal{N}=2$ superconformal theories $\quad 12$

3.1 Superconformal tensor and $\boldsymbol{O}(\mathbf{2 n})$ multiplets . . . . . . . . . . . . . 12

3.2 Superconformal polar multiplets . . . . . . . . . . . . . . . . 13

4 Reduction to $\mathcal{N}=1$ superfields

$4.1 \mathcal{N}=\mathbf{1}$ decomposition of $\mathcal{N}=\mathbf{2}$ superconformal Killings . . . . . . . . . 15

$4.2 \mathcal{N}=1$ superconformal transformations . . . . . . . . . . . . . . 16

4.3 Extended superconformal transformations . . . . . . . . . . . . 17

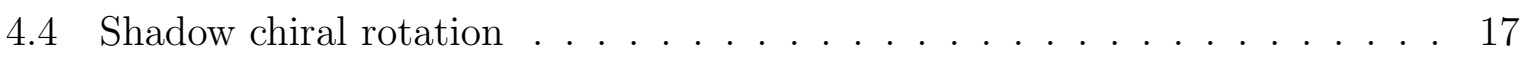

5 Non-superconformal case: $\mathcal{N}=2$ sigma-models on tangent bundles of Kähler manifolds

5.1 Background material on $\mathcal{N}=2$ sigma-models . . . . . . . . . . . . . . 19

5.2 Putting the extended supersymmetry to work . . . . . . . . . . . 20

\begin{tabular}{ll|l|}
6 & Back to the superconformal case & 24
\end{tabular}

\begin{tabular}{l|l|} 
A $\mathcal{N}$-extended superconformal Killing vectors & 25 \\
\hline
\end{tabular} 


\section{Introduction}

Hyperkähler manifolds are known to be the target spaces for systems of $4 \mathrm{D} \mathcal{N}=2$ hypermultiplets in the case of rigid supersymmetry [1]. In local supersymmetry, when the hypermultiplets couple to $\mathcal{N}=2$ supergravity, their target spaces have to be quaternionic Kähler [2]. Unlike Kähler metrics, both hyperkähler and quaternionic Kähler metrics are difficult to construct explicitly. However, the results of [1, 2] imply that the existence of regular (i.e. superspace) techniques for formulating supersymmetric nonlinear sigma-models with eight supercharges should be equivalent to a formalism to generate hyperkähler and quaternionic Kähler metrics. This idea was one of the driving motivations in the 1980 s to look for $4 \mathrm{D} \mathcal{N}=2$ off-shell supersymmetric techniques, and the latter quest has resulted in the creation of two powerful paradigms 11 harmonic superspace [3, 4] and projective superspace 2] [5, 6, 7]. The projective superspace approach is ideally suited for explicit construction of hyperkähler metrics.

Remarkably, the problem of constructing arbitrary quaternionic Kähler metrics is equivalent to that of generating hyperkähler metrics with special properties. As shown first by Swann [12] (see also [13]), there exist a one-to-one correspondence between $4 n$ dimensional quaternionic Kähler spaces and 4(n+1)-dimensional hyperkähler manifolds possessing a homothetic Killing vector (implying the fact that the isometry group includes a subgroup $\mathrm{SU}(2)$ that rotates the three complex structures). In the physics literature, such hyperkähler spaces are known as "hyperkähler cones" [15], and they turn out to be the target spaces for $4 \mathrm{D} \mathcal{N}=2$ superconformal sigma-models (see [14, 15, 16] and references therein). Given a $4(n+1)$-dimensional hyperkähler cone, the corresponding $4 n$-dimensional quaternionic Kähler space is obtained by implementing the Swann reduction [12, 13. At the sigma-model level, this was elaborated in detail in [15] 3

Thus, to generate arbitrary hyperkähler cones, it is sufficient to construct all possible superconformal sigma-models described in terms of various off-shell realizations of the 4D $\mathcal{N}=2$ massless scalar multiplet. So far, this has thoroughly been elaborated [15, 16] for only the simplest off-shell realization $-\mathcal{N}=2$ tensor multiplet [18, 19, 20] (see [21] for a

\footnotetext{
${ }^{1}$ It was Rosly [9] who first realized, building on earlier ideas due to Witten [10, that the right superspace setting for $4 \mathrm{D} \mathcal{N}=2$ supersymmetric theories is isotwistor superspace $\mathbb{R}^{4 \mid 8} \times \mathbb{C} P^{1}=\mathbb{R}^{4 \mid 8} \times S^{2}$ (following the terminology of [11]). This superspace is called "harmonic" or "projective," depending on the following two prerequisites: (i) the supermultiplets selected to inhabit it; and (ii) the supersymmetric action principle chosen.

${ }^{2}$ See 8 for a related construction in two dimensions.

${ }^{3}$ For the construction of quaternionic Kähler metrics from harmonic superspace, see [4, 17] and references therein.
} 
detailed study of tensor multiplets in $\mathcal{N}=2$ supergravity). General couplings for $\mathcal{N}=2$ tensor multiplets were actually given in the foundational work on projective superspace [5], and even earlier in [22]. As is known, the use of tensor multiplets allows one to generate very restrictive couplings. At the same time, the most interesting multiplet in projective superspace is the so-called polar multiplet [6, 7, 23], for it is believed to allow the most general sigma-model couplings 4 [6, 7]. The superconformal description of polar multiplets, as well as general superconformal couplings for polar multiplets, have been given only recently in the context of five-dimensional $\mathcal{N}=1$ supersymmetry [25]. The present paper is aimed, in part, at extending the results of [25] to four dimensions 5 ]

The main thrust of this paper is actually to address the following technical issue. When realized in terms of $\mathcal{N}=1$ superfields, the polar multiplets involve an infinite tale of auxiliary unconstrained superfields, along with two physical superfields. In nonlinear sigma-models, elimination of the auxiliaries requires solving an infinite set of algebraic nonlinear equations, and this is hard. We are going to demonstrate that this nontrivial problem can completely be avoided by making use of powerful supersymmetry considerations. Conceptually, this will be similar to the recent analysis given for the $\mathcal{N}=2$ supersymmetirc sigma-models on tangent bundles on Hermitian symmetric spaces [27].

This paper is organized as follows. In section 2 , we start by recalling the $4 \mathrm{D} \mathcal{N}=2$ superconformal kinematics, and then continue on to define superconformal projective multiplets and give several important examples. The superconformal action principle is also discussed. Superconformal sigma-models are presented in section 3. As an illustration, here we review the models for tensor (and, more generally, $O(2 n)$ ) multiplets, which have already appeared in the literature. A new family of superconformal sigma-models for polar hypermultiplets is introduced. The latter theories provide general superconformal sigma-model couplings in projective superspace. In section 4 , we discuss the reduction of $\mathcal{N}=2$ superconformal multiplets to $\mathcal{N}=1$ superfields. In section 5 , we consider a large class of polar hypermultiplet theories, which include the superconformal sigma-models as a subclass, and address the problem of eliminating the auxiliary degrees of freedom. The specific features of the superconformal sigma-models are analyzed in section 6 . Finally, some facts about $\mathcal{N}$-extended superconformal Killing vectors are collected in the appendix.

\footnotetext{
${ }^{4}$ The polar multiplet is the projective-superspace analogue of the $q^{+}$-hypermultiplet in harmonic superspace [3], see 24] for a detailed discussion of the relationship between these two approaches.

${ }^{5} \mathrm{Such}$ an extension is very natural. But since the $5 \mathrm{D}$ superspace notation and the corresponding superconformal algebar $\mathrm{F}(4)$ [26], which were use in [25], are somewhat exotic, the $4 \mathrm{D} \mathcal{N}=2$ implications of the results in [25] do not seem to be transparent even for some experts.
} 


\section{$24 \mathrm{D} \mathcal{N}=2$ superconformal formalism}

In this section, we introduce various superconformal projective multiplets and discuss the manifestly superconformal action principle. We start by recalling the key points of the superconformal formalism in $4 \mathrm{D} \mathcal{N}=2$ superspace $\mathbb{R}^{4 \mid 8}$ parametrized by coordinates $z^{A}=\left(x^{a}, \theta_{i}^{\alpha}, \bar{\theta}_{\dot{\alpha}}^{i}\right)$, where $i=\underline{1}, \underline{2}$.

\subsection{Superconformal Killing vectors}

Here we build on the formalism developed in [37] (see also [36]). By definition, a superconformal Killing vector 6

$$
\xi=\bar{\xi}=\xi^{A}(z) D_{A}=\xi^{a}(z) \partial_{a}+\xi_{i}^{\alpha}(z) D_{\alpha}^{i}+\bar{\xi}_{\dot{\alpha}}^{i}(z) \bar{D}_{i}^{\dot{\alpha}}
$$

obeys the condition

$$
\bar{D}_{\dot{\alpha}}^{i} \Phi=0 \quad \longrightarrow \quad \bar{D}_{\dot{\alpha}}^{i}(\xi \Phi)=0
$$

for an arbitrary chiral superfield $\Phi$. This condition implies the fulfillment of eq. (A.3) and also

$$
\left[\xi, D_{\alpha}^{i}\right]=-\left(D_{\alpha}^{i} \xi_{j}^{\beta}\right) D_{\beta}^{j}=\omega_{\alpha}^{\beta} D_{\beta}^{i}-\bar{\sigma} D_{\alpha}^{i}-\Lambda_{j}^{i} D_{\alpha}^{j} .
$$

The latter relation corresponds to the choice $\mathcal{N}=2$ in eq. (A.5). The parameters of (z-dependent) Lorentz $\omega$ and scale-chiral $\sigma$ transformations are

$$
\omega_{\alpha \beta}(z)=-\frac{1}{2} D_{(\alpha}^{i} \xi_{\beta) i}, \quad \sigma(z)=\frac{1}{4} \bar{D}_{i}^{\dot{\alpha}} \bar{\xi}_{\dot{\alpha}}^{i}
$$

and they can be seen to be chiral

$$
\bar{D}_{i}^{\dot{\alpha}} \omega_{\alpha \beta}=0, \quad \bar{D}_{i}^{\dot{\alpha}} \sigma=0
$$

The parameters $\Lambda_{j}^{i}$ defined by

$$
\begin{gathered}
\Lambda_{j}^{i}(z)=\frac{1}{2}\left(D_{\alpha}^{i} \xi_{j}^{\alpha}-\frac{1}{2} \delta_{j}^{i} D_{\alpha}^{k} \xi_{k}^{\alpha}\right)=-\frac{1}{2}\left(\bar{D}_{j}^{\dot{\alpha} \bar{\xi}_{\dot{\alpha}}^{i}}-\frac{1}{2} \delta_{j}^{i} \bar{D}_{k}^{\dot{\alpha} \bar{\xi}_{\dot{\alpha}}^{k}}\right) \\
\Lambda^{i j}=\Lambda^{j i}, \quad \overline{\Lambda^{i j}}=\Lambda_{i j}
\end{gathered}
$$

correspond to $\mathrm{SU}(2)$ transformations. One can readily check the identity

$$
D_{\alpha}^{k} \Lambda_{j}^{i}=-2\left(\delta_{j}^{k} D_{\alpha}^{i}-\frac{1}{2} \delta_{j}^{i} D_{\alpha}^{k}\right) \sigma
$$

\footnotetext{
${ }^{6}$ The concept of superconformal Killing vectors [28, 29, 30, 31, 32, 33, 34, is extremely useful for various studies of superconformal theories in four, five and six dimensions, see e.g. [35, 36, 37, 25.
} 
and therefore

$$
D_{\alpha}^{(i} \Lambda^{j k)}=\bar{D}_{\dot{\alpha}}^{(i} \Lambda^{j k)}=0
$$

A primary superfield $\mathcal{H}(z)$ (with its Lorentz and $\mathrm{SU}(2)$ indices suppressed) is defined to possess the superconformal transformation

$$
\delta \mathcal{H}=-\left(\xi+\omega_{\alpha}{ }^{\beta} M_{\beta}{ }^{\alpha}+\bar{\omega}_{\dot{\alpha}}{ }^{\dot{\beta}} \bar{M}_{\dot{\beta}}{ }^{\dot{\alpha}}+\Lambda_{i}{ }^{j} R_{j}{ }^{i}+2(p \sigma+q \bar{\sigma})\right) \mathcal{H}
$$

Here $M_{\alpha}{ }^{\beta}$ and $\bar{M}_{\dot{\alpha}} \dot{\beta}$ are the Lorentz generators, and $R_{j}^{i}$ the generators of $\mathrm{SU}(2)$. The parameters $p$ and $q$ determine the dimension $(p+q)$ of the superfield and its $\mathrm{U}(1)_{R}$ charge proportional to $(p-q)$.

Following [9, 3, 5], it is robust to make use of an isotwistor $u^{+i} \in \mathbb{C}^{2} \backslash\{0\}$ that allows one to introduce a subset of strictly anti-commuting spinor covariant derivatives, in accrodance with (A.4),

$$
D_{\alpha}^{+}=D_{\alpha}^{i} u_{i}^{+}, \quad \bar{D}_{\dot{\alpha}}^{+}=\bar{D}_{\dot{\alpha}}^{i} u_{i}^{+} \quad\left\{D_{\alpha}^{+}, D_{\beta}^{+}\right\}=\left\{\bar{D}_{\dot{\alpha}}^{+}, \bar{D}_{\dot{\beta}}^{+}\right\}=\left\{D_{\alpha}^{+}, \bar{D}_{\dot{\beta}}^{+}\right\}=0 .
$$

Hence, one can define so-called analytic superfield\& 7 constrained by $D_{\alpha}^{+} Q=\bar{D}_{\dot{\alpha}}^{+} Q=0$.

Let us introduce

$$
\Lambda^{++}=\Lambda^{i j} u_{i}^{+} u_{j}^{+}
$$

It follows from (2.8) that $\Lambda^{++}$is analytic,

$$
D_{\alpha}^{+} \Lambda^{++}=\bar{D}_{\dot{\alpha}}^{+} \Lambda^{++}=0
$$

In addition to $u_{i}^{+}$, it is also useful to introduce an auxiliary isotwistor $u_{i}^{-}$obeying the only condition

$$
\left(u^{+} u^{-}\right)=u^{+i} u_{i}^{-} \neq 0
$$

Of course, with $u_{i}^{-}$fixed, this condition is satisfied only on an open subset of the isotwistor space $\mathbb{C}^{2} \backslash\{0\}$. With its aid, we introduce the isotwistor derivatives (compare with [3])

$$
D^{++}=u^{+i} \frac{\partial}{\partial u^{-i}}, \quad D^{--}=u^{-i} \frac{\partial}{\partial u^{+i}}
$$

and the spinor covariant derivatives

$$
D_{\alpha}^{-}=\left[D^{--}, D_{\alpha}^{+}\right], \quad \bar{D}_{\dot{\alpha}}^{-}=\left[D^{--}, \bar{D}_{\dot{\alpha}}^{+}\right] .
$$

\footnotetext{
${ }^{7}$ Such superfields were called isochiral in [11.
} 
Since $u_{i}^{+}$and $u_{i}^{-}$form a linearly independent basis for $\mathbb{C}^{2}$, the superconformal Killing vector can also be represented as follows:

$$
\xi=\bar{\xi}=\xi^{a}(z) \partial_{a}-\frac{1}{\left(u^{+} u^{-}\right)}\left(\xi^{+\alpha} D_{\alpha}^{-}+\bar{\xi}^{+\dot{\alpha}} \bar{D}_{\dot{\alpha}}^{-}\right)+\frac{1}{\left(u^{+} u^{-}\right)}\left(\xi^{-\alpha} D_{\alpha}^{+}+\bar{\xi}^{-\dot{\alpha}} \bar{D}_{\dot{\alpha}}^{+}\right),
$$

with $\xi^{ \pm \alpha}=\xi^{\alpha i} u_{i}^{+}$and $\bar{\xi}^{+\dot{\alpha}}=\bar{\xi}^{\dot{\alpha} i} u_{i}^{+}$.

Using eq. (2.7) one can show that the following combination

$$
\Sigma=\frac{\Lambda^{+-}}{\left(u^{+} u^{-}\right)}+\sigma+\bar{\sigma}, \quad \Lambda^{+-}=\Lambda^{i j} u_{i}^{+} u_{j}^{-}
$$

possesses the properties

$$
D_{\alpha}^{+} \Sigma=\bar{D}_{\dot{\alpha}}^{+} \Sigma=0, \quad D^{++} \Sigma=\frac{\Lambda^{++}}{\left(u^{+} u^{-}\right)},
$$

and thus $\Sigma$ is analytic 8 Now, the (supervolume-preservation) identity (see, e.g. [39])

$$
(-1)^{A} D_{A} \xi^{A}=0
$$

can be rewritten in the form

$$
\partial_{a} \xi^{a}+\frac{1}{\left(u^{+} u^{-}\right)}\left(D_{\alpha}^{-} \xi^{+\alpha}+\bar{D}_{\dot{\alpha}}^{-\bar{\xi}^{+\dot{\alpha}}}-D^{--} \Lambda^{++}\right)=2 \Sigma .
$$

Eq. (2.3) implies

$$
\delta D_{\alpha}^{+} \equiv\left[\xi-\frac{\Lambda^{++}}{\left(u^{+} u^{-}\right)} D^{--}, D_{\alpha}^{+}\right]=\omega_{\alpha}^{\beta} D_{\beta}^{+}-\left(\sigma+\frac{\Lambda^{+-}}{\left(u^{+} u^{-}\right)} D_{\alpha}^{+}\right),
$$

and similarly for $\delta \bar{D}_{\dot{\alpha}}^{+}$.

\subsection{Superconformal projective multiplets: Definition}

In defining $4 \mathrm{D} \mathcal{N}=2$ superconformal multiplets in projective superspace, we closely follow the formulation of 5D superconformal off-shell multiplets given in [25], and the subsequent extension for 5D $\mathcal{N}=1$ AdS superspace [40].

A superconformal projective multiplet of weight $n, Q^{(n)}\left(z, u^{+}\right)$, is a superfield that lives on $\mathbb{R}^{4 / 8}$, is holomorphic with respect to the isotwistor variables $u_{i}^{+}$on an open domain of

\footnotetext{
${ }^{8}$ There are natural analogs of $\Lambda^{++}$and $\Sigma$ in the harmonic-superspace approach [38, 4].
} 
$\mathbb{C}^{2} \backslash\{0\}$, and is characterized by the following conditions:

(i) it obeys the analyticity constraints

$$
D_{\alpha}^{+} Q^{(n)}=\bar{D}_{\dot{\alpha}}^{+} Q^{(n)}=0
$$

(ii) it is a homogeneous function of $u^{+}$of degree $n$, that is

$$
Q^{(n)}\left(z, c u^{+}\right)=c^{n} Q^{(n)}\left(z, u^{+}\right), \quad c \in \mathbb{C}^{*}
$$

(iii) it possesses the superconformal transformation law:

$$
\delta Q^{(n)}=-\left(\xi-\frac{\Lambda^{++}}{\left(u^{+} u^{-}\right)} D^{--}\right) Q^{(n)}-n \Sigma Q^{(n)} .
$$

As a consequence of eqs. (2.18) and (2.21), the variation $\delta Q^{(n)}$ is analytic. By construction, $Q^{(n)}$ is independent of the auxiliary isotwistor $u_{i}^{-}$,

$$
\frac{\partial}{\partial u^{-i}} Q^{(n)}=0 \quad \longrightarrow \quad D^{++} Q^{(n)}=0
$$

Eq. (2.23) implies that $\delta Q^{(n)}$ is also independent of $u^{-}$,

$$
\frac{\partial}{\partial u^{-i}} \delta Q^{(n)}=0
$$

although separate contributions to the right-hand side of (2.24) involve $u^{-}$. In order for eq. (2.23) (and also eq. (2.32) ) to be unambiguous, in what follows we restrict the weight $n$ to be integer.

Using the natural projection $\pi: \mathbb{C}^{2} \backslash\{0\} \rightarrow \mathbb{C} P^{1}$, the superconformal projective multiplets can be reformulated as tensor fields that live in $\mathbb{R}^{4 \mid 8} \times \mathbb{C} P^{1}$ and are holomorphic on an open domain of $\mathbb{C} P^{1}$, see below. In the harmonic-superspace approach [3, 4], one has to deal with smooth tensor fields on $\mathbb{R}^{4 \mid 8} \times S^{2}$ which are globally defined on $S^{2}$. The projective-superspace action [5, 43] does not require the Lagrangian (and, hence, the matter superfields appearing in the Lagrangian) to be globally defined over $\mathbb{C} P^{1}$. In practice this often gives some more freedom, say, for sigma-model building.

Simplest superconformal projective multiplets are homogeneous polynomials in $u^{+}$

$$
H^{(n)}(z, u)=u_{i_{1}}^{+} \cdots u_{i_{n}}^{+} H^{i_{1} \cdots i_{n}}(z) .
$$

Following the terminology of [23], they will be called $O(n)$ multiplets. Such multiplets are globally defined on $\mathbb{C}^{2} \backslash\{0\}$. The analyticity constraints $(2.22)$ are equivalent to

$$
D_{\alpha}^{(j} H^{\left.i_{1} \cdots i_{n}\right)}=\bar{D}_{\dot{\alpha}}^{(j} H^{\left.i_{1} \cdots i_{n}\right)}=0 .
$$


The transformation law (2.24) is equivalent to

$$
\delta H^{i_{1} \cdots i_{n}}=-\xi H^{i_{1} \cdots i_{n}}-\sum_{k=1}^{n} \Lambda_{j}^{i_{k}} H^{j i_{1} \cdots \hat{i}_{k} \cdots i_{n}}-n(\sigma+\bar{\sigma}) H^{i_{1} \cdots i_{n}}
$$

where the notation $\widehat{i_{k}}$ means that the corresponding index is missing. The latter transformation law is uniquely determined by the constraints (2.28). It should be pointed out that the case $n=1$ corresponds to an on-shell Fayet-Sohnius hypermultiplet [41], $n=2$ to an off-shell tensor multiplet [19]. In the super-Poincaré case, general $O(n)$ multiplets, with $n>2$, were studied in [42, 46, 7].

The complex conjugate of an analytic superfield $Q^{(n)}$ is not analytic. However, one can introduce a generalized, analyticity-preserving conjugation $[9,3,5], Q^{(n)} \rightarrow \widetilde{Q}^{(n)}$, defined as (see also [40])

$$
\widetilde{Q}^{(n)}\left(u^{+}\right) \equiv \bar{Q}^{(n)}\left(\widetilde{u}^{+}\right), \quad \widetilde{u}^{+}=\mathrm{i} \sigma_{2} u^{+}
$$

with $\bar{Q}^{(n)}$ the complex conjugate of $Q^{(n)}$. Its fundamental property is

$$
\widetilde{D_{\alpha}^{+} Q^{(n)}}=-(-1)^{\epsilon\left(Q^{(n)}\right)} \bar{D}_{\dot{\alpha}}^{+} \widetilde{Q}^{(n)}, \quad \widetilde{\bar{D}_{\dot{\alpha}}^{+} Q^{(n)}}=(-1)^{\epsilon\left(Q^{(n)}\right)} D_{\alpha}^{+} \widetilde{Q}^{(n)}
$$

One can show

$$
\widetilde{\widetilde{Q}}^{(n)}=(-1)^{n} Q^{(n)}
$$

and therefore real supermultiplets can be consistently defined when $n$ is even. In what follows, $\widetilde{Q}^{(n)}$ will be called the smile-conjugate of $Q^{(n)}$.

By smile-conjugating the transformation law (2.24), one can see that $\widetilde{Q}^{(n)}$ is a superconformal projective multiplet of weight $n$.

\subsection{Superconformal projective multiplets: Examples}

Consider the natural projection $\pi: \mathbb{C}^{2} \backslash\{0\} \rightarrow \mathbb{C} P^{1}$. The isotwistor variables $u_{i}^{+}$ provide homogeneous global coordinates for points in $\mathbb{C} P^{1}$. Thus, any analytic superfield corresponds to a supermultiplet living in $\mathbb{R}^{4 \mid 8} \times \mathbb{C} P^{1}$. Instead of $u_{i}^{+}$, it is often useful to deal with an inhomogeneous complex coordinate $\zeta$ which is defined locally and is invariant under projective rescalings $u_{i}^{+} \rightarrow c u_{i}^{+}$, with $c \in \mathbb{C}^{*}$. Then, one should replace $Q^{(n)}\left(z, u^{+}\right)$with a new superfield $Q^{[n]}(z, \zeta) \propto Q^{(n)}\left(z, u^{+}\right)$, where $Q^{[n]}(z, \zeta)$ is holomorphic with respect to $\zeta$. As is demonstrated below, the precise definition of $Q^{[n]}(z, \zeta)$ depends on the projective supermultiplet under consideration. It is standard to cover $\mathbb{C}^{2} \backslash\{0\}$ by two open charts: (i) the north chart characterized by $u^{+1} \neq 0$; (ii) the south chart with 
$u^{+\underline{2}} \neq 0$. In discussing various supermultiplets, our consideration below will be restricted to the north chart.

Since $u^{+\underline{1}} \neq 0$ in the north chart, it is natural to introduce a projective-invariant complex variable $\zeta \in \mathbb{C}$ as follows:

$$
u^{+i}=u^{+1}(1, \zeta)=u^{+1} \zeta^{i}, \quad \zeta^{i}=(1, \zeta), \quad \zeta_{i}=\varepsilon_{i j} \zeta^{j}=(-\zeta, 1) .
$$

Any projective multiplet $Q^{(n)}$ and its superconformal variation (2.24) do not depend on $u^{-}$, and thus we can make a convenient choice for the later. It is useful to choose

$$
u_{i}^{-}=(1,0), \quad u^{-i}=\varepsilon^{i j} u_{j}^{-}=(0,-1) .
$$

For the analytic transformation parameters $\Lambda^{++}(2.11)$ and $\Sigma(2.17)$, we then have

$$
\begin{aligned}
\Lambda^{++} & =\left(u^{+\underline{1}}\right)^{2} \Lambda^{++}(\zeta), & \Lambda^{++}(\zeta) & =\Lambda^{i j} \zeta_{i} \zeta_{j}=\Lambda^{\underline{11}} \zeta^{2}-2 \Lambda^{\underline{12}} \zeta+\Lambda^{\underline{22}}, \\
\Sigma & =\Sigma(\zeta), & \Sigma(\zeta) & =\Lambda^{\underline{1} i} \zeta_{i}+\sigma+\bar{\sigma}=-\Lambda^{\underline{11}} \zeta+\Lambda^{\underline{12}}+\sigma+\bar{\sigma} .
\end{aligned}
$$

An arctic multiplet9 9 of weight $n$ is defined to be holomorphic on the north chart. It can be represented as

$$
\Upsilon^{(n)}(z, u)=\left(u^{+}\right)^{n} \Upsilon^{[n]}(z, \zeta), \quad \Upsilon^{[n]}(z, \zeta)=\sum_{k=0}^{\infty} \Upsilon_{k}(z) \zeta^{k}
$$

The superconformal transformation law of $\Upsilon^{[n]}$ can be derived from eq. (2.24) to be

$$
\delta \Upsilon^{[n]}=-\left(\xi+\Lambda^{++}(\zeta) \partial_{\zeta}\right) \Upsilon^{[n]}-n \Sigma(\zeta) \Upsilon^{[n]}
$$

This transformation law is analogous to that given in [25] in five dimensions.

The smile-conjugate of $\Upsilon^{(n)}$ is said to be an antarctic multiplet of weight $n$. It proves to be holomorphic on the south chart, while in the north chart it has the form

$$
\begin{aligned}
& \widetilde{\Upsilon}^{(n)}(z, u)=\left(u^{+2}\right)^{n} \widetilde{\Upsilon}^{[n]}(z, \zeta)=\left(u^{+}\right)^{n} \zeta^{n} \widetilde{\Upsilon}^{[n]}(z, \zeta) \\
& \widetilde{\Upsilon}^{[n]}(z, \zeta)=\sum_{k=0}^{\infty}(-1)^{k} \bar{\Upsilon}_{k}(z) \frac{1}{\zeta^{k}},
\end{aligned}
$$

with $\bar{\Upsilon}_{k}$ the complex conjugate of $U_{k}$. In accordance with (2.24), its superconformal transformation is as follows (compare with the $5 \mathrm{D}$ case [25]):

$$
\delta \widetilde{\Upsilon}^{[n]}=-\frac{1}{\zeta^{n}}\left(\xi+\Lambda^{++}(\zeta) \partial_{\zeta}\right)\left(\zeta^{n} \widetilde{\Upsilon}^{(n)}\right)-n \Sigma(\zeta) \widetilde{\Upsilon}^{(n)}
$$

\footnotetext{
${ }^{9}$ We use the terminology introduced in 23 . for various projective multiplets in the super-Poincaré case.
} 
The arctic multiplet $\Upsilon^{[n]}$ and its smile-conjugate $\widetilde{\Upsilon}^{(n)}$ constitute a polar multiplet.

In the case of a real $O(2 n)$ multiplet, it can be represented as

$$
\begin{aligned}
H^{(2 n)}\left(z, u^{+}\right) & =\left(\mathrm{i} u^{+\underline{1}} u^{+2}\right)^{n} H^{[2 n]}(z, \zeta)=\left(u^{+\underline{1}}\right)^{2 n}(\mathrm{i} \zeta)^{n} H^{[2 n]}(z, \zeta), \\
H^{[2 n]}(z, \zeta) & =\sum_{k=-n}^{n} H_{k}(z) \zeta^{k}, \quad \bar{H}_{k}=(-1)^{k} H_{-k} .
\end{aligned}
$$

In accordance with (2.24), the superconformal transformation of $H^{[2 n]}$ is

$$
\delta H^{[2 n]}=-\frac{1}{\zeta^{n}}\left(\xi+\Lambda^{++}(\zeta) \partial_{\zeta}\right)\left(\zeta^{n} H^{[2 n]}\right)-2 n \Sigma(\zeta) H^{[2 n]}
$$

analogous to the five-dimensional transformation law [25]. In a similar way one can introduce complex $O(2 n+1)$ multiplets.

Finally, let us consider a real tropical multiplet of weight $2 n$.

$$
\begin{aligned}
U^{(2 n)}\left(z, u^{+}\right) & =\left(\mathrm{i} u^{+\underline{1}} u^{+2}\right)^{n} U^{[2 n]}(z, \zeta)=\left(u^{+1}\right)^{2 n}(\mathrm{i} \zeta)^{n} U^{[2 n]}(z, \zeta) \\
U^{[2 n]}(z, \zeta) & =\sum_{k=-\infty}^{\infty} U_{k}(z) \zeta^{k}, \quad \bar{U}_{k}=(-1)^{k} U_{-k} .
\end{aligned}
$$

Its superconformal transformation copies (2.41). The case $n=1$ corresponds to supersymmetric Lagrangians, see below. A tropical multiplet with $n=0$ is used to describe the prepotential for a massless vector multiplet.

In terms of the superfield $Q^{[n]}(z, \zeta)$, the analyticity condition (2.22) takes the form

$$
D_{\alpha}^{2} Q^{[n]}(\zeta)=\zeta D_{\alpha}^{1} Q^{[n]}(\zeta), \quad \bar{D}_{\underline{2}}^{\dot{\alpha}} Q^{[n]}(\zeta)=-\frac{1}{\zeta} \bar{D}_{\underline{1}}^{\dot{\alpha}} Q^{[n]}(\zeta)
$$

This relation implies that the dependence of the component superfields $Q_{k}$ of $Q^{[n]}(\zeta)$,

$$
Q^{[n]}(z, \zeta)=\sum_{k=-\infty}^{\infty} Q_{k}(z) \zeta^{k}
$$

on $\theta_{\underline{2}}^{\alpha}$ and $\bar{\theta}_{\dot{\alpha}}^{2}$ is uniquely determined in terms of their dependence on $\theta_{\underline{1}}^{\alpha} \equiv \theta^{\alpha}$ and $\bar{\theta} \frac{1}{\dot{\alpha}} \equiv$ $\bar{\theta}_{\dot{\alpha}}$. In other words, the projective superfields depend effectively on half the Grassmann variables which can be choosen to be the spinor coordinates of $4 \mathrm{D}, \mathcal{N}=1$ superspace. If the series (2.44) terminates from below, then the two lowest components are constrained $\mathcal{N}=1$ superfields. In particular, in the case of the arctic multiplet (2.36), $\Phi:=\Upsilon_{0} \mid$ is chiral, $\bar{D}_{\dot{\alpha}} \Phi=0$, and $\Sigma:=\Upsilon_{1} \mid$ is complex linear, $\bar{D}^{2} \Sigma=0$. 


\subsection{Superconformal action}

Let $L^{++}\left(z, u^{+}\right) \equiv L^{(2)}\left(z, u^{+}\right)$be a real superconformal projective multiplet of weight two. Following [25], we are going to demonstrate that the action functiona 10

$$
S=\frac{1}{2 \pi} \oint \frac{u_{i}^{+} \mathrm{d} u^{+i}}{\left(u^{+} u^{-}\right)^{4}} \int \mathrm{d}^{4} x\left(D^{-}\right)^{4} L^{++}\left(z, u^{+}\right) \|, \quad\left(D^{-}\right)^{4}=\frac{1}{16}\left(D^{-}\right)^{2}\left(\bar{D}^{-}\right)^{2}
$$

is invariant under arbitrary superconformal transformations. Here the line integral is carried out over a closed contour, $\gamma=\left\{u_{i}^{+}(t)\right\}$, in the space of $u^{+}$variables. The integrand in (2.45) involves a constant (i.e. $t$-independent) isotwistor $u_{i}^{-}$subject to the only condition that $u^{+}(t)$ and $u^{-}$form a linearly independent basis at each point of the contour $\gamma$, that is, eq. (2.13) holds at each point of the contour.

In (2.45), the double-bar notation, $U \|$, denotes the $\theta$-independent component of a $\mathcal{N}=2$ superfield $U\left(x, \theta_{i}, \bar{\theta}^{i}\right)$. Below, we will also a single-bar notation, $U \mid$, to denote the $\mathcal{N}=1$ projection of $U$. Thus

$$
U \|=\left.U\left(x, \theta_{i}, \bar{\theta}^{i}\right)\right|_{\theta_{i}=\bar{\theta}^{i}=0}, \quad U\left|=U\left(x, \theta_{i}, \bar{\theta}^{i}\right)\right|_{\theta_{\underline{2}}=\bar{\theta} \underline{2}=0} .
$$

Action (2.45) is invariant under arbitrary projective transformations of the form

$$
\left(u_{i}^{-}, u_{i}^{+}\right) \rightarrow\left(u_{i}^{-}, u_{i}^{+}\right) R, \quad R=\left(\begin{array}{ll}
a & 0 \\
b & c
\end{array}\right) \in \operatorname{GL}(2, \mathbb{C}) .
$$

This gauge-like symmetry implies that the action is actually independent of $u_{i}^{-}$. Using the representation (2.16) along with the analyticity conditions, the transformation law (2.24) with $n=2$ gives

$$
\delta L^{++}=-\left\{\xi^{a} \partial_{a}-\frac{1}{\left(u^{+} u^{-}\right)}\left(\xi^{+\alpha} D_{\alpha}^{-}+\bar{\xi}^{+\dot{\alpha}} \bar{D}_{\dot{\alpha}}^{-}+\Lambda^{++} D^{--}\right)\right\} L^{++}-2 \Sigma L^{++} .
$$

Making here use of eq. (2.20) leads to

$$
\begin{aligned}
\delta L^{++}=-\partial_{a}\left(\xi^{a} L^{++}\right) & -\frac{1}{\left(u^{+} u^{-}\right)}\left\{D_{\alpha}^{-}\left(\xi^{+\alpha} L^{++}\right)+\bar{D}_{\dot{\alpha}}^{-}\left(\bar{\xi}^{+\dot{\alpha}} L^{++}\right)\right\} \\
& +\frac{1}{\left(u^{+} u^{-}\right)} D^{--}\left(\Lambda^{++} L^{++}\right) .
\end{aligned}
$$

It remains to note the idenity (see [40] for a related discussion)

$$
\frac{\left(\dot{u}^{+} u^{+}\right)}{\left(u^{+} u^{-}\right)^{5}} D^{--}\left(\Lambda^{++} L^{++}\right)=-\frac{\mathrm{d}}{\mathrm{d} t}\left(\frac{\Lambda^{++} L^{++}}{\left(u^{+} u^{-}\right)^{4}}\right),
$$

\footnotetext{
${ }^{10}$ In the super-Poincaré case, this action was introduced in [5]. It was re-formulated in a manifestly projective-invariant form in [43].
} 
where $\left(\dot{u}^{+} u^{+}\right) \mathrm{d} t=u_{i}^{+} \mathrm{d} u^{+i}$ is part of the line integral measure in (2.45). Since the line integral in (2.45) corresponds to a closed contour, the action is seen to be invariant.

We can now formulate a general superconformal Lagrangian:

$$
L^{++}\left(z, u^{+}\right)=L\left(Q^{(n)}\left(z, u^{+}\right)\right), \quad L\left(Q^{(n)}\left(z, c u^{+}\right)\right)=c^{2} L\left(Q^{(n)}\left(z, u^{+}\right)\right) .
$$

Here the dynamical variables $Q^{(n)}$ are superconformal projective multiplets.

\subsection{Projective gauge fixing}

Without loss of generality, one can assume that the integration contour in (2.45) does not pass through the "north pole" $u^{+i} \sim(0,1)$. Then, one can introduce the complex variable $\zeta$ as in (2.33), and fix the projective invariance (2.47) as in (2.34). If we also represent the Lagrangian in the form

$$
L^{++}\left(z, u^{+}\right)=\mathrm{i} u^{+1} u^{+2} L(z, \zeta)=\mathrm{i}\left(u^{+1}\right)^{2} \zeta L(z, \zeta),
$$

the action reduces to

$$
S=\frac{1}{16} \oint \frac{\mathrm{d} \zeta}{2 \pi \mathrm{i}} \int \mathrm{d}^{4} x \zeta\left(D^{\underline{1}}\right)^{2}\left(\bar{D}_{\underline{2}}\right)^{2} L(z, \zeta) \|
$$

Finally, making use of the analyticity of $L$ gives

$$
S=\frac{1}{2 \pi \mathrm{i}} \oint \frac{\mathrm{d} \zeta}{\zeta} \int \mathrm{d}^{4} x \mathrm{~d}^{4} \theta L(z, \zeta) \mid
$$

where the integration is carried out over the $\mathcal{N}=1$ superspace.

The Lagrangian $L(z, \zeta)$ introduced in $(2.52)$ is characterized by the following superconformal transformation:

$$
-\zeta \delta L=\partial_{a}\left(\xi^{a} \zeta L\right)+D_{\alpha}^{-}\left(\xi^{+\alpha} \zeta L\right)+\bar{D}_{\dot{\alpha}}^{-}\left(\bar{\xi}^{+\dot{\alpha}} \zeta L\right)+\partial_{\zeta}\left(\Lambda^{++}(\zeta) \zeta L\right) .
$$

It makes obvious the superconformal invariance of (2.53). Eq. (2.55) can be compared with the five-dimensional transformation in [25].

\section{$34 \mathrm{D} \mathcal{N}=2$ superconformal theories}

\subsection{Superconformal tensor and $O(2 n)$ multiplets}

Superconformal self-couplings of tensor multiplets are well-known [5, 15]. For a set of tensor multiplets $H^{++I}$, with $I=1, \ldots, n$, superconformal dynamics is generated by 
a Lagrangian $L^{++}=L\left(H^{++I}\right)$ that is a real homogeneous function of first degree in the variables $H^{++}$,

$$
H^{++I} \frac{\partial}{\partial H^{++I}} L\left(H^{++}\right)=L\left(H^{++}\right)
$$

Generalizations for $O(2 n)$ multilets are obvious.

To describe the improved $\mathcal{N}=2$ tensor multiplet [44] in projective superspace, some special considerations are required. But since such a formulation is well-known [5, 45, 15], we will not discuss it here.

We should point out that some examples of superconformal self-couplings for tensor and $O(4)$ multiplets in harmonic superspace were given in [46] and [47] respectively.

\subsection{Superconformal polar multiplets}

We consider a system of interacting arctic weight-one multiplets $\Upsilon^{+}\left(z, u^{+}\right)$and their smile-conjugates $\widetilde{\Upsilon}^{+}$described by the Lagrangian [25, 40]

$$
L^{++}=\mathrm{i} K\left(\Upsilon^{+}, \widetilde{\Upsilon}^{+}\right)
$$

with $K\left(\Phi^{I}, \bar{\Phi}^{\bar{J}}\right)$ a real analytic function of $n$ complex variables $\Phi^{I}$, where $I=1, \ldots, n$. Since $L^{++}=L^{++}\left(z, u^{+}\right)$is required to be a weight-two projective superfield, the potential $K$ has to respect the following homogeneity condition

$$
\left(\Phi^{I} \frac{\partial}{\partial \Phi^{I}}+\bar{\Phi}^{\bar{I}} \frac{\partial}{\partial \bar{\Phi}^{\bar{I}}}\right) K(\Phi, \bar{\Phi})=2 K(\Phi, \bar{\Phi})
$$

For $L^{++}$to be real, we require a stronger condition

$$
\Phi^{I} \frac{\partial}{\partial \Phi^{I}} K(\Phi, \bar{\Phi})=K(\Phi, \bar{\Phi})
$$

Then, representing $\Upsilon^{+}\left(z, u^{+}\right)=u^{+1} \Upsilon(z, \zeta)$ and $\widetilde{\Upsilon}^{+}\left(z, u^{+}\right)=u^{+2} \tilde{\Upsilon}(z, \zeta)$, we can rewrite the Lagrangian in the form

$$
L^{++}\left(z, u^{+}\right)=\mathrm{i} u^{+1} u^{+2} L(z, \zeta), \quad L=K(\Upsilon, \widetilde{\Upsilon})
$$

The action takes the form

$$
S=\frac{1}{2 \pi \mathrm{i}} \oint \frac{\mathrm{d} \zeta}{\zeta} \int \mathrm{d}^{4} x \mathrm{~d}^{4} \theta K\left(\Upsilon^{I}, \widetilde{\Upsilon}^{\bar{J}}\right),
$$


with the integration contour around the origin in $\mathbb{C}$. We should emphasise that action (3.6) is formulated in terms of $\mathcal{N}=1$ superfields, but it is invariant under linearly realized $\mathcal{N}=2$ superconformal transformations.

There is a simple algebraic construction to generate superconformal actions of the form (3.6). Let $\mathcal{P}_{n}\left(w^{a}\right)=\mathcal{P}_{n}\left(w^{1}, \ldots, w^{q}\right)$ be a homogeneous polynomial of order $n$ in $q$ complex variables $w^{a}, \mathcal{P}_{n}\left(c w^{a}\right)=c^{n} \mathcal{P}_{n}\left(w^{a}\right)$. Given a constant Hermitian matrix $\eta_{\bar{a} b}$, we consider the action

$$
S=\frac{1}{2 \pi \mathrm{i}} \oint \frac{\mathrm{d} \zeta}{\zeta} \int \mathrm{d}^{4} x \mathrm{~d}^{4} \theta \widetilde{\Upsilon}^{\bar{a}} \eta_{\bar{a} b} \Upsilon^{b}
$$

with the weight-one arctic multiplets $\Upsilon^{a}(z, \zeta)$ obeying the constraint

$$
\mathcal{P}_{n}\left(\Upsilon^{a}\right)=0
$$

Suppose that the dynamical variables $\Upsilon^{I}(z, \zeta)$ in (3.6) include a compensator $\Upsilon(z, \zeta)$, that is an arctic multiplet such that its lowest-order ( $\zeta$-independent) component $\Upsilon_{0}$ is everywhere non-vanishing. Then, we can introduce new dynamical variables comprising the unique weight-one multiplet $\Upsilon(z, \zeta)$ and some set of weight-zero arctic multiplets $v^{i}(z, \zeta)$. The action (3.6) will then turn into

$$
S=\frac{1}{2 \pi \mathrm{i}} \oint \frac{\mathrm{d} \zeta}{\zeta} \int \mathrm{d}^{4} x \mathrm{~d}^{4} \theta \widetilde{\Upsilon} \Upsilon \mathrm{e}^{\mathcal{K}(v, \widetilde{v})},
$$

with $\mathcal{K}(v, \widetilde{v})$ a Kähler potential. This action is invariant under Kähler tansformations

$$
\Upsilon \longrightarrow \mathrm{e}^{-\Lambda(v)} \Upsilon, \quad \mathcal{K}(v, \widetilde{v}) \rightarrow \mathcal{K}(v, \widetilde{v})+\Lambda(v)+\bar{\Lambda}(\widetilde{v})
$$

with $\Lambda$ a holomorphic function. Action (3.9) is reminiscent of that describing a general chiral sigma-model in $4 \mathrm{D} \mathcal{N}=1$ old minimal supergravity provided one switches off the gravitational superfield $H^{m}$ and keeps only the chiral compensator $\varphi$ alive (see, e. g., [32] for a review), with the latter being replaced with $\Upsilon$ in the $\mathcal{N}=2$ case.

\section{Reduction to $\mathcal{N}=1$ superfields}

The important powerful feature of the projective supermultiplets is that they admit a simple decomposition in terms of standard $\mathcal{N}=1$ superfields. In the superconformal case, it is therefore useful to reduce the $\mathcal{N}=2$ superconformal transformation laws of the projective supermultiplets to $\mathcal{N}=1$ superfields. This is explicitly carried out in the present section. 


\section{1 $\mathcal{N}=1$ decomposition of $\mathcal{N}=2$ superconformal Killings}

It turns out that the $\mathcal{N}=2$ superconformal Killing vector $\xi$ generates three types of transformations at the level of $\mathcal{N}=1$ superfields. In terms of the $\mathcal{N}=1$ projection

$$
\begin{aligned}
\xi \mid & :=\xi^{A} \mid D_{A}, \\
{\left[\xi \mid, D_{\alpha}^{\frac{1}{\alpha}}\right] } & =\omega_{\alpha}^{\beta}\left|D_{\beta}^{\frac{1}{\beta}}-\left(\bar{\sigma}\left|+\Lambda_{\underline{1}}\right|\right) D_{\alpha}^{\underline{1}}-\Lambda_{\underline{2}} \underline{\underline{1}}\right| D_{\alpha}^{\underline{2}}
\end{aligned}
$$

they are as follows:

1. An arbitrary $\mathcal{N}=1$ superconformal transformation generated by

$$
\boldsymbol{\xi}=\overline{\boldsymbol{\xi}}=\boldsymbol{\xi}^{a}(z) \partial_{a}+\boldsymbol{\xi}^{\alpha}(z) D_{\alpha}+\overline{\boldsymbol{\xi}}_{\dot{\alpha}}(z) \bar{D}^{\dot{\alpha}}
$$

such that

$$
\left[\boldsymbol{\xi}, D_{\alpha}^{i}\right]=\boldsymbol{\omega}_{\alpha}^{\beta} D_{\beta}+(\boldsymbol{\sigma}-2 \overline{\boldsymbol{\sigma}}) D_{\alpha},
$$

see the appendix. The components of $\boldsymbol{\xi}$ and their descendants $\boldsymbol{\omega}_{\alpha}{ }^{\beta}$ and $\boldsymbol{\sigma}$ correspond to the following choice of the parameters in (4.1):

$$
\xi\left|=\boldsymbol{\xi}, \quad \omega_{\alpha}^{\beta}\right|=\boldsymbol{\omega}_{\alpha}^{\beta}, \quad \sigma\left|=\boldsymbol{\sigma}, \quad \Lambda_{\underline{1}} \underline{1}\right|=\overline{\boldsymbol{\sigma}}-\boldsymbol{\sigma}, \quad \Lambda_{\underline{2}} \underline{\underline{1}} \mid=0 .
$$

2. An extended superconformal transformation generated by

$$
\begin{aligned}
\xi \mid & =\rho^{\alpha} D_{\alpha}^{2}+\bar{\rho}_{\dot{\alpha}} \bar{D}_{\underline{2}}^{\dot{\alpha}}, & & \xi_{\underline{2}}^{\alpha} \mid=\rho^{\alpha}, \\
\omega_{\alpha}^{\beta} \mid & =\sigma\left|=\Lambda_{\underline{1}} \underline{\underline{1}}\right|=0, & & \Lambda_{\underline{2}} \underline{\underline{1}}\left|=\Lambda^{\underline{11}}\right|=-\frac{1}{2} D^{\alpha} \rho_{\alpha} .
\end{aligned}
$$

3. A shadow chiral rotation. This is a phase transformation of $\theta_{\underline{2}}^{\alpha}$ only, with $\theta_{\underline{1}}^{\alpha}$ kept unchanged, and it corresponds to the choice

$$
\xi\left|=0, \quad \omega_{\alpha}^{\beta}\right|=\Lambda_{\underline{2}} \underline{\underline{1}}|=0, \quad \sigma|=\Lambda_{\underline{1}} \underline{\underline{1}}|=-\bar{\sigma}|=-\frac{\mathrm{i}}{2} \alpha .
$$

The spinor parameter $\rho^{\alpha}$ in (4.5) can be shown to obey the equations

$$
\bar{D}_{\dot{\alpha}} \rho^{\beta}=0, \quad D^{(\alpha} \rho^{\beta)}=0,
$$

and the latter imply

$$
\partial^{\dot{\alpha}(\alpha} \rho^{\beta)}=D^{2} \rho^{\beta}=0
$$

There are several ordinary (component) transformations generated by the chiral spinor $\rho^{\alpha}$ in (4.5): (i) second Q-supersymmetry transformation $\left(\epsilon^{\alpha}\right)$; (ii) off-diagonal SU(2)transformation $\left(\lambda=\left.\Lambda^{\underline{11}}\right|_{\theta=0}\right)$; (iii) second S-supersymmetry transformation $\left(\bar{\eta}_{\dot{\alpha}}\right)$. They emerge as follows: $\rho^{\alpha}\left(x_{(+)}, \theta\right)=\epsilon^{\alpha}+\lambda \theta^{\alpha}-\mathrm{i} \bar{\eta}_{\dot{\alpha}} x_{(+)}^{\dot{\alpha} \alpha}$, with $x_{(+)}^{a}$ the chiral extension of $x^{a}$. 


\section{$4.2 \mathcal{N}=1$ superconformal transformations}

Let us first consider how the $\mathcal{N}=2$ superconformal multiplets vary under the $\mathcal{N}=1$ superconformal transformations described by eqs. (4.2 - 4.4). Here the superconfomal building blocks (2.35) take the form:

$$
\Lambda^{++}(\zeta)|=2 \zeta(\overline{\boldsymbol{\sigma}}-\boldsymbol{\sigma}), \quad \Sigma(\zeta)|=2 \boldsymbol{\sigma} .
$$

Consider the arctic multiplet of weight $n$, eq. (2.36). Its $\mathcal{N}=2$ superconformal transformation law (2.37) implies

$$
\delta \Upsilon_{k}=-\boldsymbol{\xi} \Upsilon_{k}-2 k(\overline{\boldsymbol{\sigma}}-\boldsymbol{\sigma}) \Upsilon_{k}-2 n \boldsymbol{\sigma} \Upsilon_{k}
$$

In particular, for the leading chiral $\Phi:=\Upsilon_{0}$ and complex linear $\Sigma:=\Upsilon_{1}$ components we get

$$
\delta \Phi=-\boldsymbol{\xi} \Phi-2 n \boldsymbol{\sigma} \Phi, \quad \delta \Sigma=-\boldsymbol{\xi} \Sigma-2 \overline{\boldsymbol{\sigma}} \Sigma-2(n-1) \boldsymbol{\sigma} \Sigma .
$$

These transformation laws can be seen to be consistent with the off-shell constraints $\bar{D}_{\dot{\alpha}} \Phi=0$ and $\bar{D}^{2} \Sigma=0$.

Consider the real $O(2 n)$ multiplet (2.41). Its $\mathcal{N}=2$ superconformal transformation law (2.41) implies

$$
\delta H_{k}=-\boldsymbol{\xi} H_{k}+2(k-n) \boldsymbol{\sigma} H_{k}-2(k+n) \overline{\boldsymbol{\sigma}} H_{k} .
$$

In particular, for the leading chiral $\Phi:=H_{-n}$ and complex linear $\Sigma:=H_{-n+1}$ components we get

$$
\delta \Phi=-\boldsymbol{\xi} \Phi-4 n \boldsymbol{\sigma} \Phi, \quad \delta \Sigma=-\boldsymbol{\xi} \Sigma-2 \overline{\boldsymbol{\sigma}} \Sigma-2(2 n-1) \boldsymbol{\sigma} \Sigma .
$$

These transformation laws are consistent with the off-shell constraints $\bar{D}_{\dot{\alpha}} \Phi=0$ and $\bar{D}^{2} \Sigma=0$. As is seen from (4.12), the variation of the real superfield $H_{0}$ is real.

For $n>1$, the real $O(2 n)$ multiplet describes an off-shell hypermultiplet. The special case $n=1$ corresponds to an off-shell tensor multiplet. In accordance with (4.13), the real linear superfield $G:=H_{0}=\bar{G}$ transforms as

$$
\delta G=-\boldsymbol{\xi} G-2(\overline{\boldsymbol{\sigma}}+\boldsymbol{\sigma}) G .
$$

This transformation law is uniquely fixed by the off-shell constraints $\bar{D}^{2} G=D^{2} G=0$. 


\subsection{Extended superconformal transformations}

We now turn to the extended superconformal transformations (4.5). In this case, the superconformal building blocks are

$$
\Lambda^{++}(\zeta)\left|=-\frac{1}{2}\left(\zeta^{2} D^{\alpha} \rho_{\alpha}+\bar{D} \dot{\alpha}^{\dot{\alpha}}\right), \quad \Sigma(\zeta)\right|=\frac{1}{2} \zeta D^{\alpha} \rho_{\alpha}
$$

To read off the corresponding transformations of the component $\mathcal{N}=1$ superfields of $\mathcal{N}=2$ multiplets, it remains to use the identity

$$
\left(\rho^{\alpha} D_{\alpha}^{2}+\bar{\rho}_{\dot{\alpha}} \bar{D}_{2}^{\dot{\alpha}}\right) Q^{[n]}(z, \zeta)=\left(\zeta \rho^{\alpha} D_{\alpha}-\frac{1}{\zeta} \bar{\rho}_{\dot{\alpha}} \bar{D}^{\dot{\alpha}}\right) Q^{[n]}(z, \zeta)
$$

that follows form the analyticity constraint.

For the arctic multiplet of weight $n$, eq. (2.36), we obtain

$$
\begin{aligned}
\delta \Upsilon_{0}= & \bar{\rho}_{\dot{\alpha}} \bar{D}^{\dot{\alpha}} \Upsilon_{1}+\frac{1}{2}\left(\bar{D}_{\dot{\alpha}} \bar{\rho}^{\dot{\alpha}}\right) \Upsilon_{1}, \\
\delta \Upsilon_{1}= & -\rho^{\alpha} D_{\alpha} \Upsilon_{0}+\bar{D}_{\dot{\alpha}}\left(\bar{\rho}^{\dot{\alpha}} \Upsilon_{2}\right)-\frac{n}{2}\left(D^{\alpha} \rho_{\alpha}\right) \Upsilon_{0}, \\
\delta \Upsilon_{k}= & -\rho^{\alpha} D_{\alpha} \Upsilon_{k-1}+\bar{\rho}_{\dot{\alpha}} \bar{D}^{\dot{\alpha}} \Upsilon_{k+1} \\
& +\frac{1}{2}(k-n-1)\left(D^{\alpha} \rho_{\alpha}\right) \Upsilon_{k-1}+\frac{1}{2}(k+1)\left(\bar{D}_{\dot{\alpha}} \bar{\rho}^{\dot{\alpha}}\right) \Upsilon_{k+1}, \quad k>1 .
\end{aligned}
$$

One can see that the transformation laws in (4.17a) are consistent with the off-shell constraints $\bar{D}_{\dot{\alpha}} \Upsilon_{0}=0$ and $\bar{D}^{2} \Upsilon_{1}=0$.

For the real $O(2 n)$ multiplet (2.41), we obtain

$$
\begin{aligned}
\delta H_{-n}= & \bar{\rho}_{\dot{\alpha}} \bar{D}^{\dot{\alpha}} H_{-n+1}+\frac{1}{2}\left(\bar{D} \dot{\alpha}^{\dot{\alpha}}\right) H_{-n+1}, \\
\delta H_{-n+1}= & -\rho^{\alpha} D_{\alpha} H_{-n}+\bar{D}_{\dot{\alpha}}\left(\bar{\rho}^{\dot{\alpha}} H_{-n+2}\right)-n\left(D^{\alpha} \rho_{\alpha}\right) H_{-n}, \\
\delta H_{k}= & -\rho^{\alpha} D_{\alpha} H_{k-1}+\bar{\rho}_{\dot{\alpha}} \bar{D}^{\dot{\alpha}} H_{k+1}-\frac{1}{2}(n+1-k)\left(D^{\alpha} \rho_{\alpha}\right) H_{k-1} \\
& +\frac{1}{2}(n+1+k)\left(\bar{D}_{\dot{\alpha}} \bar{\rho}^{\dot{\alpha}}\right) H_{k+1}, \quad-n+1<k<0, \\
\delta H_{0}= & -\rho^{\alpha} D_{\alpha} H_{-1}-\bar{\rho}_{\dot{\alpha}} \bar{D}^{\dot{\alpha}} \bar{H}_{-1}-\frac{1}{2}(n+1)\left(\left(D^{\alpha} \rho_{\alpha}\right) H_{-1}+\left(\bar{D}_{\dot{\alpha}} \bar{\rho}^{\dot{\alpha}}\right) \bar{H}_{-1}\right) .
\end{aligned}
$$

\subsection{Shadow chiral rotation}

Finally, let us consider the shadow chiral rotation (4.6). In the case of the arctic multiplet of weight $n$, eq. (2.36), it acts as follows:

$$
\delta \Upsilon_{k}=\mathrm{i} \alpha\left(k-\frac{n}{2}\right) \Upsilon_{k}
$$


For the real $O(2 n)$ multiplet (2.41), we obtain

$$
\delta H_{k}=\mathrm{i} \alpha k H_{k} .
$$

The component $H_{0}$ is real, and therefore it does not transform. In a finite form, this transformation reads

$$
\begin{aligned}
& \Upsilon(z, \zeta) \quad \longrightarrow \quad \Upsilon^{\prime}(z, \zeta)=\mathrm{e}^{-\mathrm{i}(n / 2) \alpha} \Upsilon\left(z, \mathrm{e}^{\mathrm{i} \alpha} \zeta\right), \\
& H^{[2 n]}(z, \zeta) \quad \longrightarrow \quad H^{[2 n] \prime}(z, \zeta)=H^{[2 n]}\left(z, \mathrm{e}^{\mathrm{i} \alpha} \zeta\right)
\end{aligned}
$$

\section{Non-superconformal case: $\mathcal{N}=2$ sigma-models on tangent bundles of Kähler manifolds}

Before turning to a analysis of the superconformal dynamical system (3.6), it is instructive to consider a more general family of $4 \mathrm{D} \mathcal{N}=2$ off-shell supersymmetric nonlinear sigma-models that are described in ordinary $\mathcal{N}=1$ superspace by the action 11

$$
S[\Upsilon, \widetilde{\Upsilon}]=\frac{1}{2 \pi \mathrm{i}} \oint \frac{\mathrm{d} \zeta}{\zeta} \int \mathrm{d}^{4} x \mathrm{~d}^{4} \theta K\left(\Upsilon^{I}(\zeta), \widetilde{\Upsilon}^{\bar{J}}(\zeta)\right)
$$

The arctic $\Upsilon(\zeta)$ and antarctic $\widetilde{\Upsilon}(\zeta)$ dynamical variables are generated by an infinite set of ordinary superfields:

$$
\Upsilon(\zeta)=\sum_{n=0}^{\infty} \Upsilon_{n} \zeta^{n}=\Phi+\Sigma \zeta+O\left(\zeta^{2}\right), \quad \widetilde{\Upsilon}(\zeta)=\sum_{n=0}^{\infty} \bar{\Upsilon}_{n}(-\zeta)^{-n}
$$

Here $\Phi$ is chiral, $\Sigma$ complex linear,

$$
\bar{D}_{\dot{\alpha}} \Phi=0, \quad \bar{D}^{2} \Sigma=0,
$$

and the remaining component superfields are unconstrained complex superfields. The above theory occurs as a minimal $\mathcal{N}=2$ extension of the general four-dimensional $\mathcal{N}=1$ supersymmetric nonlinear sigma-model [48]

$$
S[\Phi, \bar{\Phi}]=\int \mathrm{d}^{4} x \mathrm{~d}^{4} \theta K\left(\Phi^{I}, \bar{\Phi}^{\bar{J}}\right)
$$

with $K$ the Kähler potential of a Kähler manifold $\mathcal{M}$.

The reason we are interested here in the $\mathcal{N}=2$ supersymmetric theory (15.1) is that its action becomes superconformal upon imposing the homogeneity condition (3.4).

\footnotetext{
${ }^{11}$ The study of such models was initiated in [24, 49, 50, and important results have recently been obtained in [51, 27]. They correspond to a subclass of the general hypermultiplet theories in projective superspace [6, 7] obtained by replacing $K(\Upsilon, \widetilde{\Upsilon}) \rightarrow K(\Upsilon, \widetilde{\Upsilon}, \zeta)$ in (5.1).
} 


\subsection{Background material on $\mathcal{N}=2$ sigma-models}

The extended supersymmetric sigma-model (5.1) inherits all the geometric features of its $\mathcal{N}=1$ predecessor (5.4). The Kähler invariance of the latter, $K(\Phi, \bar{\Phi}) \rightarrow K(\Phi, \bar{\Phi})+$ $\Lambda(\Phi)+\bar{\Lambda}(\bar{\Phi})$, turns into

$$
K(\Upsilon, \widetilde{\Upsilon}) \quad \longrightarrow \quad K(\Upsilon, \widetilde{\Upsilon})+\Lambda(\Upsilon)+\bar{\Lambda}(\widetilde{\Upsilon})
$$

for the model (5.1) 12 A holomorphic reparametrization of the Kähler manifold, $\Phi^{I} \rightarrow$ $\Phi^{\prime I}=f^{I}(\Phi)$, has the following counterpart

$$
\Upsilon^{I}(\zeta) \quad \longrightarrow \quad \Upsilon^{\prime I}(\zeta)=f^{I}(\Upsilon(\zeta))
$$

in the $\mathcal{N}=2$ case. Therefore, the physical superfields of the $\mathcal{N}=2$ theory

$$
\left.\Upsilon^{I}(\zeta)\right|_{\zeta=0}=\Phi^{I},\left.\quad \frac{\mathrm{d} \Upsilon^{I}(\zeta)}{\mathrm{d} \zeta}\right|_{\zeta=0}=\Sigma^{I}
$$

should be regarded, respectively, as coordinates of a point in the Kähler manifold and a tangent vector at the same point. Thus the variables $\left(\Phi^{I}, \Sigma^{J}\right)$ parametrize the tangent bundle $T \mathcal{M}$ of the Kähler manifold $\mathcal{M}[25]$.

To describe the theory in terms of the physical superfields $\Phi$ and $\Sigma$ only, all the auxiliary superfields have to be eliminated with the aid of the corresponding algebraic equations of motion

$$
\oint \frac{\mathrm{d} \zeta}{\zeta} \zeta^{n} \frac{\partial K(\Upsilon, \widetilde{\Upsilon})}{\partial \Upsilon^{I}}=\oint \frac{\mathrm{d} \zeta}{\zeta} \zeta^{-n} \frac{\partial K(\Upsilon, \widetilde{\Upsilon})}{\partial \widetilde{\Upsilon}^{\bar{J}}}=0, \quad n \geq 2 .
$$

Let $\Upsilon_{*}(\zeta) \equiv \Upsilon_{*}(\zeta ; \Phi, \bar{\Phi}, \Sigma, \bar{\Sigma})$ denote a unique solution subject to the initial conditions

$$
\Upsilon_{*}(0)=\Phi, \quad \dot{\Upsilon}_{*}(0)=\Sigma
$$

For a general Kähler manifold $\mathcal{M}$, the auxiliary superfields $\Upsilon_{2}, \Upsilon_{3}, \ldots$, and their conjugates, can be eliminated only perturbatively. Their elimination can be carried out using the ansatz 52 ]

$$
\Upsilon_{n}^{I}=\sum_{p=0}^{\infty} G_{J_{1} \ldots J_{n+p} \bar{L}_{1} \ldots \bar{L}_{p}}^{I}(\Phi, \bar{\Phi}) \Sigma^{J_{1}} \ldots \Sigma^{J_{n+p}} \bar{\Sigma}^{\bar{L}_{1}} \ldots \bar{\Sigma}^{\bar{L}_{p}}, \quad n \geq 2
$$

\footnotetext{
${ }^{12}$ In the superconfomal case, the Lagrangian obeys the homogeneity condition (3.4), and no Kähler invariance survives.
} 
Assuming that the auxiliary superfields have been eliminated, the action (5.1) should take the form 13 [49, 50]:

$$
\begin{aligned}
S_{\mathrm{tb}}[\Phi, \Sigma] & =\frac{1}{2 \pi \mathrm{i}} \oint \frac{\mathrm{d} \zeta}{\zeta} \int \mathrm{d}^{4} x \mathrm{~d}^{4} \theta K\left(\Upsilon_{*}(\zeta), \breve{\Upsilon}_{*}(\zeta)\right) \\
& =\int \mathrm{d}^{4} x \mathrm{~d}^{4} \theta\{K(\Phi, \bar{\Phi})+\mathcal{L}(\Phi, \bar{\Phi}, \Sigma, \bar{\Sigma})\} \\
\mathcal{L} & =\sum_{n=1}^{\infty}(-1)^{n} \mathcal{L}_{I_{1} \cdots I_{n} \bar{J}_{1} \ldots \bar{J}_{n}}(\Phi, \bar{\Phi}) \Sigma^{I_{1}} \ldots \Sigma^{I_{n}} \bar{\Sigma}^{\bar{J}_{1}} \ldots \bar{\Sigma}^{\bar{J}_{n}}:=\sum_{n=1}^{\infty}(-1)^{n} \mathcal{L}^{(n)}
\end{aligned}
$$

where $\mathcal{L}_{I \bar{J}}=g_{I \bar{J}}(\Phi, \bar{\Phi})$ and the series coefficients $\mathcal{L}_{I_{1} \cdots I_{n} \bar{J}_{1} \cdots \bar{J}_{n}}$, for $n>1$, are tensor functions of the Kähler metric $g_{I \bar{J}}(\Phi, \bar{\Phi})=\partial_{I} \partial_{\bar{J}} K(\Phi, \bar{\Phi})$, the Riemann curvature $R_{I \bar{J} K \bar{L}}(\Phi, \bar{\Phi})$ and its covariant derivatives. Each term in the action contains equal powers of $\Sigma$ and $\bar{\Sigma}$, since the original model (5.1) is invariant under rigid $U(1)$ transformations 14 [49]

$$
\Upsilon(\zeta) \mapsto \Upsilon\left(\mathrm{e}^{\mathrm{i} \alpha} \zeta\right) \Longleftrightarrow \Upsilon_{n}(z) \mapsto \mathrm{e}^{\mathrm{i} n \alpha} \Upsilon_{n}(z)
$$

\subsection{Putting the extended supersymmetry to work}

In the recent work [27, it was demonstrated that supersymmetry considerations allow one to avoid the problem of solving the auxiliary field equations (5.8) in the case of Hermitian symmetric spaces which possess a covariantly constant curvature tensor.

$$
\nabla_{L} R_{I_{1} \bar{J}_{1} I_{2} \bar{J}_{2}}=\bar{\nabla}_{\bar{L}} R_{I_{1} \bar{J}_{1} I_{2} \bar{J}_{2}}=0
$$

Here we address the general case of an arbitrary Kähler manifold, with no pretense of completeness.

The theory under consideration, eq. (5.1), is $\mathcal{N}=2$ super-Poincaré invariant. In terms of the superconformal formalism presented in section 2, its symmetry structure is described by those transformations which are characterised by

$$
\Lambda^{i j}=\sigma=0
$$

These conditions correspond to the $\mathcal{N}=2$ Killing supervectors. In particular, the parameter $\rho^{\alpha}$ in (4.5) should be restricted to be a constant spinor, $\rho^{\alpha}=\varepsilon^{\alpha}=$ const. Then,

\footnotetext{
${ }^{13}$ As compared with the expressions in [49, [50, the series for $\mathcal{L}$ contains an extra factor of $(-1)^{n}$. The reason for its insertion will become clear in next subsection.

${ }^{14}$ Transformation (5.12) coincides with the shadow chiral rotation (4.21) for $n=0$.
} 
the arctic multiplet transformation laws (4.17a) and (4.17b) become

$$
\begin{aligned}
& \delta \Upsilon_{0}=\bar{\varepsilon}_{\dot{\alpha}} \bar{D}^{\dot{\alpha}} \Upsilon_{1}, \quad \delta \Upsilon_{1}=-\varepsilon^{\alpha} D_{\alpha} \Upsilon_{0}+\bar{\varepsilon}_{\dot{\alpha}} \bar{D}^{\dot{\alpha}} \Upsilon_{2}, \\
& \delta \Upsilon_{k}=-\varepsilon^{\alpha} D_{\alpha} \Upsilon_{k-1}+\bar{\varepsilon}_{\dot{\alpha}} \bar{D}^{\dot{\alpha}} \Upsilon_{k+1}, \quad k>1 .
\end{aligned}
$$

Upon elimination of the auxiliary superfields, the action (5.11) should be invariant under the supersymmetry transformations

$$
\delta \Phi=\bar{\varepsilon}_{\dot{\alpha}} \bar{D}^{\dot{\alpha}} \Sigma, \quad \delta \Sigma=-\varepsilon^{\alpha} D_{\alpha} \Phi+\bar{\varepsilon}_{\dot{\alpha}} \bar{D}^{\dot{\alpha}} \Upsilon_{2}(\Phi, \bar{\Phi}, \Sigma, \bar{\Sigma})
$$

where $\Upsilon_{2}$ now a composite field of the general form given in (5.10). Since $\Upsilon_{2}$ transforms as a connection under the holomorphic reparametrizations (5.6)

$$
\Upsilon_{2}^{I} \quad \longrightarrow \quad \Upsilon_{2}^{\prime I}=\frac{1}{2} \frac{\partial^{2} f^{I}(\Phi)}{\partial \Phi^{J} \partial \Phi^{K}} \Sigma^{J} \Sigma^{K}+\frac{\partial f^{I}(\Phi)}{\partial \Phi^{J}} \Sigma^{J}
$$

we can rewrite $\Upsilon_{2}$ in a slightly more specific form:

$$
\begin{aligned}
\Upsilon_{2}^{I} & =-\frac{1}{2} \Gamma_{J K}^{I}(\Phi, \bar{\Phi}) \Sigma^{J} \Sigma^{K}+\sum_{p=1}^{\infty} G_{J_{1} \ldots J_{p+2}}^{I} \bar{L}_{1} \ldots \bar{L}_{p}(\Phi, \bar{\Phi}) \Sigma^{J_{1}} \ldots \Sigma^{J_{p+2}} \bar{\Sigma}^{\bar{L}_{1}} \ldots \bar{\Sigma}^{\bar{L}_{p}}, \\
& :=-\frac{1}{2} \Gamma_{J K}^{I}(\Phi, \bar{\Phi}) \Sigma^{J} \Sigma^{K}+\sum_{p=1}^{\infty} G_{(p)}^{I},
\end{aligned}
$$

with $\Gamma_{J K}^{I}(\Phi, \bar{\Phi})$ the Christoffel symbols for the Kähler metric $g_{I \bar{J}}(\Phi, \bar{\Phi})$. Here the coefficients $G^{I}{ }_{J_{1} \ldots J_{p+2}} \bar{L}_{1} \ldots \bar{L}_{p}(\Phi, \bar{\Phi})$ are tensor functions of the Kähler metric, the Riemann curvature $R_{I \bar{J} K \bar{L}}(\Phi, \bar{\Phi})$ and its covariant derivatives.

Of course, the tensor fields $\mathcal{L}_{I_{1} \cdots I_{n} \bar{J}_{1} \ldots \bar{J}_{n}}$ in (5.11) and $G^{I}{ }_{J_{1} \ldots J_{p+2}} \bar{L}_{1 \ldots} \ldots \bar{L}_{p}$ in (5.18) are uniquely determined, in the theory with action (5.1), once (i) we have solved the auxiliary field equations (5.8); and (ii) have done the contour integral in the first line of (5.11). However, these two problems are tremendous in general. There is an alternative approach. We can look for a $\mathcal{N}=1$ supersymmetric theory of the form (5.11), which is required to be invariant under extended supersymmetric transformations (5.16) such that $\Upsilon_{2}^{I}$ is of the general form (5.18) . It is clear, from the previous considerations, that the requirement of extended supersymmetry should uniquely determine both sets of the coefficient functions $\mathcal{L}_{I_{1} \cdots I_{n} \bar{J}_{1} \cdots \bar{J}_{n}}$ and $G^{I}{ }_{J_{1} \ldots J_{p+2}} \bar{L}_{1} \ldots \bar{L}_{p}$. And it does indeed, as can be explicitly checked in leading orders of perturbation theory. Here are some low-order results:

$$
\begin{aligned}
\mathcal{L}^{(1)} & =g_{I \bar{J}} \Sigma^{I} \bar{\Sigma}^{\bar{J}}, \\
\mathcal{L}^{(2)} & =\frac{1}{4} R_{I_{1} \bar{J}_{1} I_{2} \bar{J}_{2}} \Sigma^{I_{1}} \Sigma^{I_{2}} \bar{\Sigma}^{\bar{J}_{1}} \bar{\Sigma}^{\bar{J}_{2}}, \\
\mathcal{L}^{(3)} & =\frac{1}{12}\left\{\frac{1}{6}\left\{\nabla_{I_{3}}, \bar{\nabla}_{\bar{J}_{3}}\right\} R_{I_{1} \bar{J}_{1} I_{2} \bar{J}_{2}}+R_{I_{1} \bar{J}_{1} I_{2}}{ }^{L} R_{L \bar{J}_{2} I_{3} \bar{J}_{3}}\right\} \Sigma^{I_{1}} \ldots \Sigma^{I_{3}} \bar{\Sigma}^{\bar{J}_{1}} \ldots \bar{\Sigma}^{\bar{J}_{3}},
\end{aligned}
$$


and

$$
G_{(1)}^{L}=\frac{1}{6} \nabla_{I_{3}} R_{I_{1} \bar{J} I_{2}}^{L} \Sigma^{I_{1}} \ldots \Sigma^{I_{3}} \bar{\Sigma}^{\bar{J}}
$$

The expressions for $\mathcal{L}^{(1)}$ and $\mathcal{L}^{(2)}$ first appeared in [25] and [49] respectively.

Before continuing on, we should recall the important notion of canonical coordinate system for Kähler manifolds that was introduced by Bochner in 1947 [54] and later used by Calabi in the 1950s [55] 15 In a neighborhood of any point $p$ of the Kähler manifold $\mathcal{M}$, holomorphic reparametrizations and Kähler transformations can be used to choose a coordinate system, with origin at $p$, in which the Kähler potential takes the form:

$$
\begin{aligned}
\boldsymbol{K}(\phi, \bar{\phi}) & =\boldsymbol{g}_{I \bar{J}} \phi^{I} \bar{\phi}^{\bar{J}}+\sum_{m, n \geq 2}^{\infty} \boldsymbol{K}^{(m, n)}(\phi, \bar{\phi}) \\
\boldsymbol{K}^{(m, n)}(\phi, \bar{\phi}) & :=\frac{1}{m ! n !} \boldsymbol{K}_{I_{1} \cdots I_{m} \bar{J}_{1} \ldots \bar{J}_{n}} \phi^{I_{1}} \ldots \phi^{I_{m}} \bar{\phi}^{\bar{J}_{1}} \ldots \bar{\phi}^{\bar{J}_{n}}
\end{aligned}
$$

In such a coordinate system, there still remains the freedom to perform linear holomorphic reparametrizations which can be used to set the metric at the origin, $p \in \mathcal{M}$, to be $\boldsymbol{g}_{I \bar{J}}=\delta_{I \bar{J}}$. The Taylor coefficients, $\boldsymbol{K}_{I_{1} \cdots I_{m} \bar{J}_{1} \cdots \bar{J}_{n}}$, in (5.21) turn out to be tensor functions of the Kähler metric, the Riemann curvature $R_{I \bar{J} K \bar{L}}$ and its covariant derivatives, all of them evaluated at the origin. In particular, one finds 16

$$
\begin{aligned}
\boldsymbol{K}^{(2,2)} & =\frac{1}{4} R_{I_{1} \bar{J}_{1} I_{2} \bar{J}_{2}} \phi^{I_{1}} \phi^{I_{2}} \bar{\phi}^{\bar{J}_{1}} \bar{\phi}^{\bar{J}_{2}}, \\
\boldsymbol{K}^{(3,2)} & =\frac{1}{12} \nabla_{I_{3}} R_{I_{1} \bar{J}_{1} I_{2} \bar{J}_{2}} \phi^{I_{1}} \phi^{I_{2}} \phi^{I_{3}} \bar{\phi}^{\bar{J}_{1}} \bar{\phi}^{\bar{J}_{2}}, \\
\boldsymbol{K}^{(4,2)} & =\frac{1}{48} \nabla_{I_{3}} \nabla_{I_{4}} R_{I_{1} \bar{J}_{1} I_{2} \bar{J}_{2}} \phi^{I_{1}} \ldots \phi^{I_{4}} \bar{\phi}^{\bar{J}_{1}} \bar{\phi}^{\bar{J}_{2}}, \\
\boldsymbol{K}^{(3,3)} & =\frac{1}{12}\left\{\frac{1}{6}\left\{\nabla_{I_{3}}, \bar{\nabla}_{\bar{J}_{3}}\right\} R_{I_{1} \bar{J}_{1} I_{2} \bar{J}_{2}}+R_{I_{1} \bar{J}_{1} I_{2}}{ }^{L} R_{L \bar{J}_{2} I_{3} \bar{J}_{3}}\right\} \phi^{I_{1}} \ldots \phi^{I_{3}} \bar{\phi}^{\bar{J}_{1}} \ldots \bar{\phi}^{\bar{J}_{3}} \\
\boldsymbol{K}^{(4,3)} & =\frac{1}{144}\left\{\bar{\nabla}_{\bar{J}_{3}} \nabla_{I_{3}} \nabla_{I_{4}} R_{I_{1} \bar{J}_{1} I_{2} \bar{J}_{2}}+6 R_{I_{3} \bar{J}_{3} I_{4}}{ }^{L} \nabla_{L} R_{I_{1} \bar{J}_{1} I_{2} \bar{J}_{2}}\right. \\
& \left.+4 R_{I_{1} \bar{J}_{1} L \bar{J}_{2}} \nabla_{I_{2}} R_{I_{3} \bar{J}_{3} I_{4}}{ }^{L}\right\} \phi^{I_{1}} \ldots \phi^{I_{4}} \bar{\phi}^{\bar{J}_{1}} \ldots \bar{\phi}^{\bar{J}_{3}} \\
& =\frac{1}{144} \boldsymbol{K}_{I_{1} \ldots I_{4} \bar{J}_{1} \ldots \bar{J}_{3}} \phi^{I_{1}} \ldots \phi^{I_{4}} \bar{\phi}^{\bar{J}_{1}} \ldots \bar{\phi}^{\bar{J}_{3}},
\end{aligned}
$$

\footnotetext{
${ }^{15}$ This coordinate system was re-discovered by supersymmetry practitioners in the 1980s under the name normal gauge [56, 57, 58,

${ }^{16}$ These results are easily derived by applying the relation $K_{I_{1} I_{2} \bar{J}_{1} \bar{J}_{2}}=R_{I_{1} \bar{J}_{1} I_{2} \bar{J}_{2}}+g_{M \bar{N}} \Gamma_{I_{1} I_{2}}^{M} \bar{\Gamma}_{\bar{J}_{1} \bar{J}_{2}}^{\bar{N}}$.
} 


$$
\begin{aligned}
\boldsymbol{K}^{(4,4)}= & \frac{1}{576}\left\{\bar{\nabla}_{\bar{J}_{4}} \boldsymbol{K}_{I_{1} \cdots I_{4} \bar{J}_{1} \cdots \bar{J}_{3}}+6 R_{I_{3} \bar{J}_{3} I_{4}}{ }^{L} \bar{\nabla}_{\bar{J}_{4}} \nabla_{L} R_{I_{1} \bar{J}_{1} I_{2} \bar{J}_{2}}\right. \\
& +4\left(\bar{\nabla}_{\bar{J}_{4}} R_{I_{1} \bar{J}_{1} L \bar{J}_{2}}\right) \nabla_{I_{2}} R_{I_{3} \bar{J}_{3} I_{4}}{ }^{L} \\
& \left.+6 R_{I_{1} \bar{J}_{1} I_{2}}{ }^{K}\left(R_{I_{3} \bar{J}_{2} I_{4}}{ }^{L} R_{K \bar{J}_{3} L \bar{J}_{4}}+2 R_{I_{3} \bar{J}_{2} K}{ }^{L} R_{I_{4} \bar{J}_{3} L \bar{J}_{4}}\right)\right\} \phi^{I_{1}} \ldots \phi^{I_{4}} \bar{\phi}^{\bar{J}_{1}} \ldots \bar{\phi}^{\bar{J}_{4}} \\
= & \frac{1}{576} \boldsymbol{K}_{I_{1} \cdots I_{4} \bar{J}_{1} \ldots \bar{J}_{4}} \phi^{I_{1}} \ldots \phi^{I_{4}} \bar{\phi}^{\bar{J}_{1}} \ldots \bar{\phi}^{\bar{J}_{4}}
\end{aligned}
$$

It is possible to rewrite $\boldsymbol{K}^{(4,4)}$ in a manifestly real form, but such an expression appears to be much longer than $(\underline{5.22 \mathrm{f}})$. The relations $(5.22 \mathrm{a} / 5.22 \mathrm{~d})$ appeared earlier in [59].

We should point out that in the literature, there exist closed-form expressions [60] for the Riemann normal coordinate expansion. It would be very interesting to obtain a similar expression for the canonical coordinate system.

The above relations hint at the fact that, for $m \neq n$, the tensor $\boldsymbol{K}_{I_{1} \cdots I_{m} \bar{J}_{1} \cdots \bar{J}_{n}}$ should be a sum of terms each of which is proportional to a (multiple) covariant derivative of the Riemann tensor. In other words,

$$
\nabla_{L} R_{I_{1} \bar{J}_{1} I_{2} \bar{J}_{2}}=\bar{\nabla}_{\bar{L}} R_{I_{1} \bar{J}_{1} I_{2} \bar{J}_{2}}=0 \quad \Longleftrightarrow \quad K^{(m, n)}=0, \quad m \neq n .
$$

Indeed, this holds in general.

If one compares the expressions for $\mathcal{L}^{(2)}$ and $\mathcal{L}^{(3)}$, eqs. (5.19b) and (5.19c), with those for $\boldsymbol{K}^{(2,2)}$ and $\boldsymbol{K}^{(3,3)}$ above, it is tempting to conclude that

$$
\mathcal{L}^{(n)}=\boldsymbol{K}^{(n, n)}(\phi \rightarrow \Sigma, \bar{\phi} \rightarrow \bar{\Sigma}) .
$$

Unfortunately, this does not hold in general, since for $n=4$ one finds

$$
\mathcal{L}^{(4)}=\left\{\frac{1}{576} \boldsymbol{K}_{I_{1} \ldots I_{4} \bar{J}_{1} \ldots \bar{J}_{4}}-\frac{1}{36}\left(\bar{\nabla}_{\bar{J}_{4}} R_{I_{1} \bar{J}_{1} L \bar{J}_{2}}\right) \nabla_{I_{2}} R_{I_{3} \bar{J}_{3} I_{4}}{ }^{L}\right\} \Sigma^{I_{1}} \ldots \Sigma^{I_{4}} \bar{\Sigma}^{\bar{J}_{1}} \ldots \bar{\Sigma}^{\bar{J}_{4}},
$$

compare with (5.22f). However, the correct statement is the following:

$$
\mathcal{L}^{(n)}=\boldsymbol{K}^{(n, n)}(\phi \rightarrow \Sigma, \bar{\phi} \rightarrow \bar{\Sigma})+(\nabla R) \text {-terms } .
$$

Here the second term on the right consists of those terms that vanish in the limit (5.13). Eq. (5.25) is one of the main results of this work.

In deriving (5.24), one has to make use of the expression for $G_{(2)}^{L}$ that appears in (5.18). It is

$$
\begin{aligned}
G_{(2) \bar{J}_{3}} & \equiv g_{\bar{J}_{3} L} G_{(2)}^{L}=G_{I_{1} \ldots I_{4} \bar{J}_{1} \bar{J}_{2} ; \bar{J}_{3}} \Sigma^{I_{1}} \ldots \Sigma^{I_{4}} \bar{\Sigma}^{\bar{J}_{1}} \bar{\Sigma}^{\bar{J}_{2}} \\
& =\frac{1}{6}\left\{\left(\nabla_{I_{4}} R_{I_{1} \bar{J}_{1} I_{2}}{ }^{L}\right) R_{L \bar{J}_{2} I_{3} \bar{J}_{3}}-\frac{1}{8} \boldsymbol{K}_{I_{1} \ldots I_{4} \bar{J}_{1} \bar{J}_{2} \bar{J}_{3}}\right\} \Sigma^{I_{1}} \ldots \Sigma^{I_{4}} \bar{\Sigma}^{\bar{J}_{1} \bar{\Sigma}^{\bar{J}_{2}} .}
\end{aligned}
$$


More generally, for any term in the series in (5.18), it should hold

$$
G_{(n) \bar{J}_{n+1}} \equiv g_{\bar{J}_{n+1} L} G_{(n)}^{L} \propto \boldsymbol{K}^{(n+2, n+1)}(\phi \rightarrow \Sigma, \bar{\phi} \rightarrow \bar{\Sigma})+(\nabla R) \text {-terms } .
$$

Let us recall that $\Upsilon_{*}(\zeta) \equiv \Upsilon_{*}(\zeta ; \Phi, \bar{\Phi}, \Sigma, \bar{\Sigma})$ denotes the unique solution to the auxiliary field equations (5.8) under the initial conditions (5.9)). We conjecture that $\Upsilon_{*}(\zeta)$ obeys the following generalised geodesic equation:

$$
\begin{aligned}
\frac{\mathrm{d}^{2} \Upsilon_{*}^{I}(\zeta)}{\mathrm{d} \zeta^{2}} & +\Gamma_{J K}^{I}\left(\Upsilon_{*}(\zeta), \bar{\Phi}\right) \frac{\mathrm{d} \Upsilon_{*}^{J}(\zeta)}{\mathrm{d} \zeta} \frac{\mathrm{d} \Upsilon_{*}^{K}(\zeta)}{\mathrm{d} \zeta} \\
& =2 \sum_{p=1}^{\infty} G_{J_{1} \ldots J_{p+2}}^{I} \bar{L}_{1} \ldots \bar{L}_{p}\left(\Upsilon_{*}, \bar{\Phi}\right) \frac{\mathrm{d} \Upsilon_{*}^{J_{1}}(\zeta)}{\mathrm{d} \zeta} \ldots \frac{\mathrm{d} \Upsilon_{*}^{J_{p+2}}(\zeta)}{\mathrm{d} \zeta} \bar{\Sigma}^{\bar{L}_{1}} \ldots \bar{\Sigma}^{\bar{L}_{p}}
\end{aligned}
$$

and is its unique solution under the same initial conditions. This equation is covariant with respect to holomorphic reparametrizations of the Kähler manifold. If the curvature tensor is covariantly constant, eq. (5.28) reduces to the geodesic equation given in [49, 50].

At the moment, we do not know the explicit structure of the derivatives terms in (5.25). We believe that a more systematic analysis of the invariance under extended supersymmetry transformations would allow one to determine these terms.

If the curvature tensor is covariantly constant, (5.13), there occur dramatic simplifications. In particular, here we obtain

$$
\begin{gathered}
\boldsymbol{K}(\phi, \bar{\phi})=\boldsymbol{g}_{I \bar{J}} \phi^{I} \bar{\phi}^{\bar{J}}+\sum_{n=2}^{\infty} \frac{1}{(n !)^{2}} \boldsymbol{K}_{I_{1} \cdots I_{n} \bar{J}_{1} \ldots \bar{J}_{n}} \phi^{I_{1}} \ldots \phi^{I_{n}} \bar{\phi}^{\bar{J}_{1}} \ldots \bar{\phi}^{\bar{J}_{n}} \\
\mathcal{L}=-g_{I \bar{J}} \Sigma^{I} \bar{\Sigma}^{\bar{J}}+\sum_{n=2}^{\infty} \frac{(-1)^{n}}{(n !)^{2}} \boldsymbol{K}_{I_{1} \cdots I_{n} \bar{J}_{1} \ldots \bar{J}_{n}} \Sigma^{I_{1}} \ldots \Sigma^{I_{n}} \bar{\Sigma}^{\bar{J}_{1}} \ldots \bar{\Sigma}^{\bar{J}_{n}}
\end{gathered}
$$

In refs. [51, 27], the sigma-model (5.1) was explicitly 'solved' for all Hermitian symmetric spaces except $E_{7} / E_{6} \times U(1)$. The above result allows one to address this case. Still, it would be very interesting to apply the scheme presented in [27] to the case of $\mathrm{E}_{7} / \mathrm{E}_{6} \times \mathrm{U}(1)$.

\section{Back to the superconformal case}

For the dynamical system (5.1), we have demonstrated that its description in terms of the physical superfields $(\Phi, \Sigma)$, eq. (5.11), can be achieved by making use of the power of $\mathcal{N}=2$ Poincare supersymmetry, without the need to solve the auxiliary field equations 
(5.8). Now we are prepared to turn to the analysis of the general superconformal sigmamodel (3.6).

The action (5.1) becomes superconformal upon imposing the homogeneity condition (3.4), and hence the symmetry group gets enhanced. In particular, the action (5.11) associated with (3.6) should be invariant under $\mathcal{N}=1$ superconformal transformations

$$
\delta \Phi=-\boldsymbol{\xi} \Phi-2 \boldsymbol{\sigma} \Phi, \quad \delta \Sigma=-\boldsymbol{\xi} \Sigma-2 \overline{\boldsymbol{\sigma}} \Sigma
$$

and extended supeconformal transformations

$$
\begin{aligned}
& \delta \Phi=\bar{\rho}_{\dot{\alpha}} \bar{D}^{\dot{\alpha}} \Sigma+\frac{1}{2}\left(\bar{D}_{\dot{\alpha}} \bar{\rho}^{\dot{\alpha}}\right) \Sigma, \\
& \delta \Sigma=-\rho^{\alpha} D_{\alpha} \Phi-\frac{n}{2}\left(D^{\alpha} \rho_{\alpha}\right) \Phi+\bar{D}_{\dot{\alpha}}\left\{\bar{\rho}^{\dot{\alpha}} \Upsilon_{2}(\Phi, \bar{\Phi}, \Sigma, \bar{\Sigma})\right\}
\end{aligned}
$$

where $\Upsilon_{2}(\Phi, \bar{\Phi}, \Sigma, \bar{\Sigma})$ is given by eq. (5.18). What are the implications of these additional symmetries? Actually it can be seen that no additional implications occur. If the action (5.11) is $\mathcal{N}=2$ supersymmetric, and the Kähler potential $K(\Phi, \bar{\Phi})$ obeys the homogeneity condition (3.4), the theory is $\mathcal{N}=2$ superconformal.

While this paper was in the process of writing-up, there appeared a new work in the archive [61], in which some superconformal aspects of $4 \mathrm{D} \mathcal{N}=2$ projective superspace were discussed, see also [25].

\section{Acknowledgements:}

Discussions with Ulf Lindström, Martin Roček and Gabriele Tartaglino-Mazzucchelli are gratefully acknowledged. It is pleasure to acknowledge hospitality of the 5th Simons Workshop where this project was largely completed. This work is supported in part by the Australian Research Council.

\section{A $\mathcal{N}$-extended superconformal Killing vectors}

In the main body of this paper, we have made extensive use of the $\mathcal{N}=1$ and $\mathcal{N}=2$ superconformal Killing vectors. Here we collect, following [36, 37], the essential information about the $\chi$-extended superconformal Killing vectors, specifically for $\mathcal{N} \leq 3$.

In $4 \mathrm{D} \mathcal{N}$-extended superspace $\mathbb{R}^{4 \mid 4 \mathcal{N}}$ parametrized by coordinates $z^{A}=\left(x^{a}, \theta_{i}^{\alpha}, \bar{\theta}_{\dot{\alpha}}^{i}\right)$, with $i=1, \ldots, \mathcal{N}$, an infinitesimal superconformal transformation $z^{A} \rightarrow z^{A}+\xi \cdot z^{A}$ is 
generated by a superconformal Killing vector

$$
\xi=\bar{\xi}=\xi^{a}(z) \partial_{a}+\xi_{i}^{\alpha}(z) D_{\alpha}^{i}+\bar{\xi}_{\dot{\alpha}}^{i}(z) \bar{D}_{i}^{\dot{\alpha}}
$$

defined to satisfy

$$
\left[\xi, \bar{D}_{i}^{\dot{\alpha}}\right] \propto \bar{D}_{j}^{\dot{\beta}}
$$

and therefore

$$
\bar{D}_{i}^{\dot{\alpha}} \xi_{j}^{\beta}=0, \quad \bar{D}_{i}^{\dot{\alpha}} \xi^{\dot{\beta} \beta}=4 \mathrm{i} \varepsilon^{\dot{\alpha} \dot{\beta}} \xi_{i}^{\beta} .
$$

The spinor covariant derivatives are assumed to obey the anti-commutation relations

$$
\left\{D_{\alpha}^{i}, D_{\beta}^{j}\right\}=\left\{\bar{D}_{\dot{\alpha} i}, \bar{D}_{\dot{\beta}_{j}}\right\}=0, \quad\left\{D_{\alpha}^{i}, \bar{D}_{\dot{\beta}_{j}}\right\}=-2 \mathrm{i}\left(\sigma^{c}\right)_{\alpha \dot{\beta}} \partial_{c}
$$

It follows from eqs. (A.2) and (A.3)

$$
\left[\xi, D_{\alpha}^{i}\right]=-\left(D_{\alpha}^{i} \xi_{j}^{\beta}\right) D_{\beta}^{j}=\omega_{\alpha}{ }^{\beta} D_{\beta}^{i}-\frac{1}{\mathcal{N}}((\mathcal{N}-2) \sigma+2 \bar{\sigma}) D_{\alpha}^{i}-\Lambda_{j}^{i} D_{\alpha}^{j} .
$$

Here the parameters of 'local' Lorentz $\omega$ and scale-chiral $\sigma$ transformations are

$$
\omega_{\alpha \beta}(z)=-\frac{1}{\mathcal{N}} D_{(\alpha}^{i} \xi_{\beta) i}, \quad \sigma(z)=\frac{1}{\mathcal{N}(\mathcal{N}-4)}\left(\frac{1}{2}(\mathcal{N}-2) D_{\alpha}^{i} \xi_{i}^{\alpha}-\bar{D}_{i}^{\dot{\alpha}} \bar{\xi}_{\dot{\alpha}}^{i}\right)
$$

and turn out to be chiral

$$
\bar{D}_{i}^{\dot{\alpha}} \omega_{\alpha \beta}=0, \quad \bar{D}_{i}^{\dot{\alpha}} \sigma=0 .
$$

The parameters $\Lambda_{j}^{i}$ defined by

$$
\Lambda_{j}{ }^{i}(z)=-\frac{\mathrm{i}}{32}\left(\left[D_{\alpha}^{i}, \bar{D}_{\dot{\alpha} j}\right]-\frac{1}{\mathcal{N}} \delta_{j}{ }_{j}\left[D_{\alpha}^{k}, \bar{D}_{\dot{\alpha} k}\right]\right) \xi^{\dot{\alpha} \alpha}, \quad \Lambda^{\dagger}=-\Lambda, \quad \operatorname{tr} \Lambda=0
$$

correspond to 'local' $S U(\mathcal{N})$ transformations. One can readily check the identity

$$
D_{\alpha}^{k} \Lambda_{j}^{i}=-2\left(\delta_{j}^{k} D_{\alpha}^{i}-\frac{1}{\mathcal{N}} \delta_{j}^{i} D_{\alpha}^{k}\right) \sigma .
$$

The explicit expressions for the components $\xi^{a}(z)$ and $\xi_{i}^{\alpha}(z)$ of an arbitrary superconformal Killing vector can be found in [25], eq. (3.15). 


\section{References}

[1] L. Alvarez-Gaumé and D. Z. Freedman, "Geometrical structure and ultraviolet finiteness in the supersymmetric sigma model," Commun. Math. Phys. 80, 443 (1981).

[2] J. Bagger and E. Witten, "Matter couplings in $\mathrm{N}=2$ supergravity," Nucl. Phys. B 222, 1 (1983).

[3] A. S. Galperin, E. A. Ivanov, S. N. Kalitsyn, V. Ogievetsky, E. Sokatchev, "Unconstrained $\mathrm{N}=2$ matter, Yang-Mills and supergravity theories in harmonic superspace," Class. Quant. Grav. 1, 469 (1984).

[4] A. S. Galperin, E. A. Ivanov, V. I. Ogievetsky and E. S. Sokatchev, Harmonic Superspace, Cambridge University Press, Cambridge, 2001.

[5] A. Karlhede, U. Lindström and M. Roček, "Self-interacting tensor multiplets in N=2 superspace," Phys. Lett. B 147, 297 (1984).

[6] U. Lindström and M. Roček, "New hyperkähler metrics and new supermultiplets," Commun. Math. Phys. 115, 21 (1988).

[7] U. Lindström and M. Roček, "N=2 super Yang-Mills theory in projective superspace," Commun. Math. Phys. 128, 191 (1990).

[8] S. J. Gates, Jr., C. M. Hull and M. Roček, "Twisted multiplets and new supersymmetric nonlinear sigma models," Nucl. Phys. B 248, 157 (1984).

[9] A. A. Rosly, "Super Yang-Mills constraints as integrability conditions," in Proceedings of the International Seminar on Group Theoretical Methods in Physics," (Zvenigorod, USSR, 1982), M. A. Markov (Ed.), Nauka, Moscow, 1983, Vol. 1, p. 263 (in Russian); English translation: in Group Theoretical Methods in Physics," M. A. Markov, V. I. Man'ko and A. E. Shabad (Eds.), Harwood Academic Publishers, London, Vol. 3, 1987, p. 587.

[10] E. Witten, "An interpretation of classical Yang-Mills theory," Phys. Lett. B 77, 394 (1978).

[11] A. A. Rosly and A. S. Schwarz, "Supersymmetry in a space with auxiliary dimensions," Commun. Math. Phys. 105, 645 (1986).

[12] A. Swann, "HyperKähler and quaternionic Kähler geometry," Math. Ann. 289, 421 (1991).

[13] K. Galicki, "Geometry of the scalar couplings in $\mathrm{N}=2$ supergravity models," Class. Quant. Grav. 9, 27 (1992).

[14] B. de Wit, B. Kleijn and S. Vandoren, "Superconformal hypermultiplets," Nucl. Phys. B 568, 475 (2000) hep-th/9909228. 
[15] B. de Wit, M. Roček and S. Vandoren, "Hypermultiplets, hyperkähler cones and quaternionKähler geometry,” JHEP 0102, 039 (2001) hep-th/0101161].

[16] M. Roček, C. Vafa and S. Vandoren, "Hypermultiplets and topological strings," JHEP 0602, 062 (2006) hep-th/0512206.

[17] E. Ivanov and G. Valent, "Quaternionic metrics from harmonic superspace: Lagrangian approach and quotient construction," Nucl. Phys. B 576, 543 (2000) hep-th/0001165.

[18] J. Wess, "Supersymmetry and internal symmetry," Acta Phys. Austriaca 41, 409 (1975).

[19] B. de Wit and J. W. van Holten, "Multiplets of linearized SO(2) supergravity," Nucl. Phys. B 155, 530 (1979); B. de Wit, J. W. van Holten and A. Van Proeyen, "Structure of N=2 supergravity," Nucl. Phys. B 184, 77 (1981) [Erratum-ibid. B 222, 516 (1983)].

[20] P. Breitenlohner and M. F. Sohnius, "Superfields, auxiliary fields, and tensor calculus for N=2 extended supergravity," Nucl. Phys. B 165, 483 (1980); M. F. Sohnius, K. S. Stelle and P. C. West, "Representations of extended supersymmetry," in Supergravity and Superspace, S. W. Hawking and M. Roček (Eds.), Cambridge Univ. Press, 1981, p. 283; P. S. Howe, K. S. Stelle and P. K. Townsend, "The relaxed hypermultiplet: An unconstrained N=2 superfield theory," Nucl. Phys. B 214, 519 (1983).

[21] B. de Wit and F. Saueressig, "Off-shell N = 2 tensor supermultiplets," JHEP 0609, 062 (2006) hep-th/0606148.

[22] U. Lindström and M. Roček, "Scalar tensor duality and N=1, N=2 nonlinear sigma models," Nucl. Phys. B 222, 285 (1983).

[23] F. Gonzalez-Rey, U. Lindström M. Roček, R. von Unge and S. Wiles, "Feynman rules in $\mathrm{N}=2$ projective superspace. I: Massless hypermultiplets," Nucl. Phys. B 516, 426 (1998) hep-th/9710250.

[24] S. M. Kuzenko, "Projective superspace as a double-punctured harmonic superspace," Int. J. Mod. Phys. A 14, 1737 (1999) hep-th/9806147.

[25] S. M. Kuzenko, "On compactified harmonic/projective superspace, 5D superconformal theories, and all that," Nucl. Phys. B 745, 176 (2006) hep-th/0601177.

[26] V. G. Kac, "Lie superalgebras," Adv. Math. 26, 8 (1977); W. Nahm, "Supersymmetries and their representations," Nucl. Phys. B 135, 149 (1978).

[27] M. Arai, S. M. Kuzenko and U. Lindström, "Polar supermultiplets, Hermitian symmetric spaces and hyperkähler metrics, 0709.2633 [hep-th]. 
[28] M. F. Sohnius, "The conformal group in superspace," in Quantum Theory and the Structures of Time and Space, Vol. 2, L. Castell , M. Drieschner and C. F. von Weizsäcker (Eds.), Carl Hanser Verlag, München, 1977, p. 241.

[29] W. Lang, "Construction of the minimal superspace translation tensor and the derivation of the supercurrent," Nucl. Phys. B 179, 106 (1981).

[30] L. Bonora, P. Pasti and M. Tonin, "Cohomologies and anomalies in supersymmetric theories," Nucl. Phys. B 252, 458 (1985).

[31] K. i. Shizuya, "Supercurrents and superconformal symmetry," Phys. Rev. D 35, 1848 (1987).

[32] I. L. Buchbinder and S. M. Kuzenko, Ideas and Methods of Supersymmetry and Supergravity or a Walk Through Superspace, IOP, Bristol, 1998.

[33] P. S. Howe and G. G. Hartwell, "A superspace survey," Class. Quant. Grav. 12, 1823 (1995).

[34] P. C. West, "Introduction to rigid supersymmetric theories," hep-th/9805055.

[35] H. Osborn, "N = 1 superconformal symmetry in four-dimensional quantum field theory," Annals Phys. 272, 243 (1999) hep-th/9808041.

[36] J. H. Park, "Superconformal symmetry in six-dimensions and its reduction to four," Nucl. Phys. B 539, 599 (1999) hep-th/9807186]; "Superconformal symmetry and correlation functions," Nucl. Phys. B 559, 455 (1999) hep-th/9903230.

[37] S. M. Kuzenko and S. Theisen, "Correlation functions of conserved currents in $\mathrm{N}=2$ superconformal theory," Class. Quant. Grav. 17, 665 (2000) hep-th/9907107.

[38] A. S. Galperin, E. A. Ivanov, V. I. Ogievetsky and E. S. Sokatchev, "Conformal invariance in harmonic superspace," in Quantum Field Theory and Quantum Statistics, I. Batalin, C. J. Isham and G. Vilkovisky (Eds.), Vol. 2, Adam Hilger, Bristol, 1987, p. 233.

[39] I. L. Buchbinder, S. M. Kuzenko and A. A. Tseytlin, "On low-energy effective actions in $\mathrm{N}=2,4$ superconformal theories in four dimensions," Phys. Rev. D 62, 045001 (2000) hep-th/9911221.

[40] S. M. Kuzenko and G. Tartaglino-Mazzucchelli, "Five-dimensional N = 1 AdS superspace: Geometry, off-shell multiplets and dynamics," Nucl. Phys. B 785, 34 (2007), 0704.1185 [hep-th].

[41] M. F. Sohnius, "Supersymmetry and central charges," Nucl. Phys. B 138 (1978) 109. 
[42] S. V. Ketov, "New self-interaction for $\mathrm{N}=2$ multiplets in $4 \mathrm{~d}$ and ultraviolet finiteness of two-dimensional N = 4 sigma-models," in Proceedings of the International Seminar Group Theory Methods in Physics, (Urmala, USSR, May 1985) Nauka, Moscow, Vol. 1, p. 87; S. V. Ketov and B. B. Lokhvitsky, "Some generalizations of N=2 Yang-Mills matter couplings," Class. Quant. Grav. 4, L137 (1987); S. V. Ketov, B. B. Lokhvitsky and I. V. Tyutin, "Hyperkahler sigma models in extended superspace," Theor. Math. Phys. 71, 496 (1987).

[43] W. Siegel, "Chiral actions for N=2 supersymmetric tensor multiplets" Phys. Lett. B 153 (1985) 51.

[44] B. de Wit, R. Philippe and A. Van Proeyen, "The improved tensor multiplet in N=2 supergravity," Nucl. Phys. B 219, 143 (1983).

[45] N. J. Hitchin, A. Karlhede, U. Lindström and M. Roček, "Hyperkähler metrics and supersymmetry," Commun. Math. Phys. 108, 535 (1987).

[46] A. Galperin, E. Ivanov and V. Ogievetsky, "Superspace actions and duality transformations for N=2 tensor multiplets," Sov. J. Nucl. Phys. 45, 157 (1987); "Duality transformations and most general matter self-couplings in $\mathrm{N}=2$ supersymmetry," Nucl. Phys. B 282, 74 (1987).

[47] S. V. Ketov, "Superconformal hypermultiplets in superspace," Nucl. Phys. B 582, 95 (2000) hep-th/0001109.

[48] B. Zumino, "Supersymmetry and Kähler manifolds," Phys. Lett. B 87, 203 (1979).

[49] S. J. Gates Jr. and S. M. Kuzenko, "The CNM-hypermultiplet nexus," Nucl. Phys. B 543, 122 (1999) hep-th/9810137.

[50] S. J. Gates Jr. and S. M. Kuzenko, "4D N = 2 supersymmetric off-shell sigma models on the cotangent bundles of Kähler manifolds," Fortsch. Phys. 48, 115 (2000) hep-th/9903013.

[51] M. Arai, S. M. Kuzenko and U. Lindström, "Hyperkähler sigma models on cotangent bundles of Hermitian symmetric spaces using projective superspace," JHEP 0702, 100 (2007) hep-th/0612174.

[52] S. M. Kuzenko and W. D. Linch, "On five-dimensional superspaces," JHEP 0602, 038 (2006) hep-th/0507176.

[53] M. Arai and M. Nitta, "Hyper-Kähler sigma models on (co)tangent bundles with SO(n) isometry," Nucl. Phys. B 745, 208 (2006) hep-th/0602277.

[54] S. Bochner, "Curvature in Hermitian metric," Bull. Amer. Math. Soc. 53, 179 (1947). 
[55] E. Calabi, "Isometric imbedding of complex manifolds," Ann. of Math., 58, 1 (1953); "On compact, locally symmetric Kähler manifolds," Ann. of Math., 71, 472 (1960).

[56] S. J. Gates, Jr., M. T. Grisaru, M. Roček and W. Siegel, Superspace, Or One Thousand and One Lessons in Supersymmetry, Benjamin/Cummings, 1983 hep-th/0108200.

[57] L. Alvarez-Gaume and P. H. Ginsparg, "Finiteness of Ricci flat supersymmetric nonlinear sigma models," Commun. Math. Phys. 102, 311 (1985).

[58] C. M. Hull, A. Karlhede, U. Lindström and M. Roček, "Nonlinear sigma models and their gauging in and out of superspace," Nucl. Phys. B 266, 1 (1986).

[59] K. Higashijima, E. Itou and M. Nitta, "Normal coordinates in Kaehler manifolds and the background field method," Prog. Theor. Phys. 108, 185 (2002) hep-th/0203081.

[60] A. D. Dolgov and I. B. Khriplovich, "Normal coordinates along a geodesic," Gen. Rel. Grav. 15, 1033 (1983); U. Müller, C. Schubert and A. M. E. van de Ven, "A closed formula for the Riemann normal coordinate expansion," Gen. Rel. Grav. 31, 1759 (1999) gr-qc/9712092.

[61] M. Hatsuda and W. Siegel, "Superconformal spaces and implications for superstrings," 0709.4605 [hep-th]. 